Portland State University

PDXScholar

6-8-2021

\title{
A Method for Comparative Analysis of Trusted Execution Environments
}

Stephano Cetola

Portland State University

Follow this and additional works at: https://pdxscholar.library.pdx.edu/open_access_etds

Part of the Computer Engineering Commons, and the Information Security Commons Let us know how access to this document benefits you.

Recommended Citation

Cetola, Stephano, "A Method for Comparative Analysis of Trusted Execution Environments" (2021). Dissertations and Theses. Paper 5720.

https://doi.org/10.15760/etd.7593

This Thesis is brought to you for free and open access. It has been accepted for inclusion in Dissertations and Theses by an authorized administrator of PDXScholar. Please contact us if we can make this document more accessible: pdxscholar@pdx.edu. 
A Method for Comparative Analysis of Trusted Execution Environments

by

Stephano Cetola

A thesis submitted in partial fulfillment of the requirements for the degree of

\author{
Master of Science \\ in \\ Electrical and Computer Engineering
}

Thesis Committee:

John M. Acken, Chair

Roy Kravitz

Tom Schubert

Portland State University

2021 
(C) 2021 Stephano Cetola

This work is licensed under a Creative Commons "Attribution 4.0 International" license.

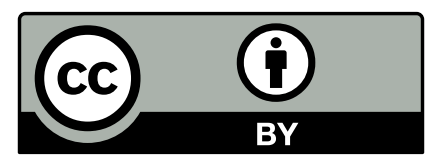




\begin{abstract}
The problem of secure remote computation has become a serious concern of hardware manufacturers and software developers alike. Trusted Execution Environments (TEEs) are a solution to the problem of secure remote computation in applications ranging from "chip and pin" financial transactions [40] to intellectual property protection in modern gaming systems [17]. While extensive literature has been published about many of these technologies, there exists no current model for comparing TEEs. This thesis provides hardware architects and designers with a set of tools for comparing TEEs. I do so by examining several properties of a TEE and comparing their implementations in several technologies. I found that several features can be detailed out into multiple sub-feature sets, which can be used in comparisons. The intent is that choosing between different technologies can be done in a rigorous way, taking into account the current features available to TEEs.
\end{abstract}




\section{Dedication}

To my wife Dana, my daughter Kristy, and my son Chad.

For all their love and support. 


\section{Acknowledgments}

I would like to thank my thesis committee, John M. Acken, Roy Kravitz, and Tom Schubert, for their guidance in writing and refining this work, as well as for their classes which helped to inspire my journey.

For their patience in teaching me the intricacies of that arcane art known as firmware, I thank Brian Richardson, Mike Kinney, and Vincent Zimmer.

For explaining countless complex computational subjects, inadvertently helping

me chose a thesis topic, and for answering my endless stream of questions over the course of many sushi dinners, I thank Brian Avery.

And my thanks to Mike Lestik, who first introduced me to Plato's Euthyphro and Newton's Calculus, and has been a lifelong friend. 


\section{Contents}

Abstract $\quad$ i

Dedication $\quad$ ii

Acknowledgments $\quad$ iii

List of Tables vi vi

List of Figures vii

Glossary viii

Acronyms $\quad x$

1 Introduction 1

2 Background 5

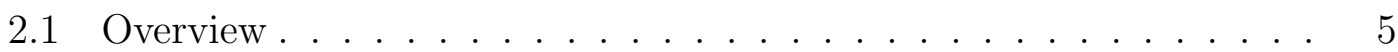

2.2 The Predecessors of the TEE . . . . . . . . . . . . 5

2.3 Birth of a TEE . . . . . . . . . . . . . . . . 8

2.4 From Handsets to the Cloud . . . . . . . . . . . . . . . . . . . . 13

2.5 RISC-V: An Open Source TEE . . . . . . . . . . . . . . . 14

3 Intel Software Guard Extensions $\quad 17$

3.1 The Intel SGX Solution . . . . . . . . . . . . . . . 17

3.2 Initial SGX Enclave Setup . . . . . . . . . . . . . . . . 17

3.3 Executing SGX Enclave Code . . . . . . . . . . . . . . . . 19 
3.4 Life Cycle of an SGX Enclave . . . . . . . . . . . . . . . . 21

3.5 Attestation with Intel SGX . . . . . . . . . . . . . . . 23

4 Arm TrustZone $\quad 27$

4.1 The Arm TrustZone Solution . . . . . . . . . . . . . . 27

4.2 Arm Trusted Firmware . . . . . . . . . . . . . . . . . . 31

4.3 TrustZone Attestation . . . . . . . . . . . . . . . . . . 34

5 RISC-V Physical Memory Protection 40

5.1 The RISC-V Open Source ISA . . . . . . . . . . . . . . . 40

5.2 The RISC-V Memory Model . . . . . . . . . . . . . . . . . . 42

5.3 RISC-V Physical Memory Attributes . . . . . . . . . . . . . . 44

5.4 RISC-V Physical Memory Protection . . . . . . . . . . . . . . 46

5.5 Keystone Enclave . . . . . . . . . . . . . . . . . . . . . . 49

5.6 Future Extensions of RISC-V Memory Protection . . . . . . . . . . 53

6 Trusted Execution Environment Comparisons 55

6.1 A Method for Comparing TEEs . . . . . . . . . . . . . 55

6.2 Mapping Data Points . . . . . . . . . . . . . . . . . 59

6.3 Considerations and Limitations . . . . . . . . . . . . . . . 64

$\begin{array}{lll}7 & \text { Conclusion } & 65\end{array}$

$\begin{array}{ll}\text { References } & 67\end{array}$ 


\section{List of Tables}

2.1 OMTP Threat Groups . . . . . . . . . . . . . . . . . 11

4.1 Arm Privilege Level Mapping . . . . . . . . . . . . . . . . . . 31

5.1 RISC-V Atomic Instructions for I/O . . . . . . . . . . . . 45

5.2 RISC-V Processor Modes . . . . . . . . . . . . . . . . . . . . 46

6.1 TEE Features and their dependencies . . . . . . . . . . . . . . . . 61

6.2 Attestation Comparison . . . . . . . . . . . . . . . . . 62

6.3 Extensibility Comparison . . . . . . . . . . . . . . . 63 


\section{List of Figures}

1.1 Protection Rings . . . . . . . . . . . . . . . . . . 2

2.1 GlobalPlatforms Typical Chipset Architecture . . . . . . . . . 12

2.2 Hardware Security Timeline . . . . . . . . . . . . . . 16

3.1 High Level SGX Overview . . . . . . . . . . . . . . . 18

3.2 Setting Up Intel SGX . . . . . . . . . . . . . . . . . . . . 19

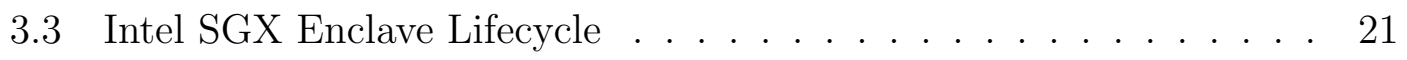

3.4 Intel SGX Attestation . . . . . . . . . . . . . . 25

4.1 High Level TrustZone Overview . . . . . . . . . . . . . . 28

4.2 High Level Overview of Trusted Board Boot . . . . . . . . . . . . 32

4.3 TrustZone Example of Normal and Secure World . . . . . . . . 34

4.4 Detail of Trusted Firmware Trusted Board Boot . . . . . . . . . . 36

4.5 OP-TEE Chain of Trust . . . . . . . . . . . . . 38

5.1 RISC-V 32 bit PMP CSR Layout and Format . . . . . . . . . 47

5.2 High Level RISC-V PMP Overview . . . . . . . . . . . . . . . 49

5.3 Keystone System Overview . . . . . . . . . . . . . . 50

5.4 Keystone PMP Protection . . . . . . . . . . . . . 51 


\section{Glossary}

attestation The process of assuring the validity and security of a system. The system can only be valid and secure if the code and data stored on the system have not been altered in any way that either causes the system to operate outside specifications or compromises the trust model. 4, 11, 14, 17, 21-25, $34,39,55-60,64$

axiomatic A semantic of logic which formalizes the definition of a concept using a set of criteria or axioms, all of which must be true for the given definition to be met. 43

chain of trust A series of entities that engage in secure transactions in order to provide a service. The first entity in the chain is referred to as the root of trust, while the last entity in the chain is often the end user or application requiring a secure transaction. $9,32,37,38$

cryptographic hash A cryptographic hash is the result of a mathematical function whose input is data of variable length and whose output is data of a deterministic value and fixed length. If this hash represents a piece of software, we can consider this hash the software's "identity" [9] for purposes of attestation. Many systems of attestation refer to this hash as a "measurement". $19,22,24,34,57$

hart In a RISC-V system, a core contains an independent instruction fetch unit and each core can have multiple hardware threads. These hardware threads are referred to as harts. 42,43 
operational semantic A semantic of logic which formalizes the definition of a concept by generating a golden output model. Any system meeting the definition must produce the same output as that defined in the model. 43

privilege ring Also known as a "protection ring" or "protection domain" [33], these modes of operation allow a processor to restrict access to memory or special instructions. Switching between rings is the function of low-level software or firmware. Before the problem of secure remote computation described in Chapter 1, these rings provided adequate protection for software applications. 1, 5, 7, 8, 19, 21-23, 30

Root of Trust "A computing engine, code, and possibly data, all co-located on the same platform which provides security services. No ancestor entity is able to provide trustworthy attestation for the initial code and data state of the Root of Trust" [25]. For system security purposes one might say, "the buck stops here". xi, 11, 17, 35, 53, 57

Trusted Compute Base When referenced generally, the code which must be trusted in order for the system to be considered secure. The code can include platform firmware, firmware from the manufacturer, or any code running inside the TEE. When in reference to a specific application, the TCB may only refer to the part of the application which runs inside the TCB. xii, 17, 57 


\section{Acronyms}

AEX Asynchronous Enclave Exit. 20

AMBA Advanced Microcontroller Bus Architecture. 28

AMO atomic memory operation. 44, 45

AXI Advanced eXtensible Interface. 28

DMA Direct Memory Access. 6, 11, 17, 54

EFI Extensible Firmware Interface. 6, 7

EPC Enclave Page Cache. 18-20, 22, 23

EPID Enhanced Privacy ID. 24, 25

ePMP Extended Physical Memory Protection. 15, 16, 53, 54

Intel ME Intel Management Engine. 6, 8, 16

IOPMP I/O Physical Memory Protection. 15, 16, 54

MSR model-specific register. 17

NMI non-maskable interrupt. 5

OMTP Open Mobile Terminal Platform. 10-12, 16

OP-TEE Open-source Portable TEE. 26, 27, 35, 37, 38, 60 
OTP One Time Programmable. 32

PCMD Paging Crypto MetaData. 23

PMA Physical Memory Attributes. 14, 44-46

PMP Physical Memory Protection. 2-4, 14-16, 39, 41, 42, 46-50, 53-55, 62, 65, 66

PRM Processor Reserved Memory. 17-19

REE Rich Execution Environment. 13, 37

RISC reduced instruction set computer. 40

RoT Root of Trust. 11, 17, 35, 36, 53, 57, 58, 62

ROTPK Root of Trust Public Key Hash. 32, 33

RVWMO RISC-V Weak Memory Order. 42-45

SCR Secure Configuration Register. 27, 29

SECS SGX Enclave Control Structure. 22

SEV Secure Encrypted Virtualization. 13

SGX Software Guard Extensions. 2-4, 8, 13-17, 19, 20, 23-28, 48, 55, 57, 60, 64

SMC Secure Monitor Call. 29, 37

SMI System Management Interrupt. 5, 6

SMM System Management Mode. 5-7, 16, 17 
SMRAM System Management RAM. 6, 7

SoC System on Chip. 11, 13, 16, 27-29, 32, 34

sPMP S-Mode Physical Memory Protection. 15, 16, 53, 54

SSA State Save Area. 20, 21

TA Trusted Application. 11, 37, 38

TBB Trusted Board Boot. 32, 33, 36

TCB Trusted Compute Base. 17, 24, 57, 58

TCG Trusted Computing Group. 9

TCS Thread Control Structure. 20

TEE Trusted Execution Environment. i, ix, 1-16, 23, 26, 28, 30, 32, 34, 37-39, $48-50,52-66$

TF-A Trusted Firmware-A. 26, 27, 31, 33, 35, 36, 38, 60

TLB Translation Lookaside Buffer. 44

TPM Trusted Platform Module. 8, 9, 12, 15-17, 38

TXT Trusted Execution Technology. 17

TZASC TrustZone Address Space Controller. 29

TZMA TrustZone Memory Adapter. 29

UEFI Unified Extensible Firmware Interface. 6, 18, 19, 33, 35 
VA Version Array. 23

VMS Virtual Machine Extension. 7

VT-x Intel Virtualization Technology. 7, 16 


\section{Chapter 1}

\section{Introduction}

Historically, computer architecture security relied on processor modes or privilege modes where code was allowed to execute. In these modes, separation of privileges is achieved and often referred to as "rings" with "ring 0" being the most privileged machine mode where OS kernel code runs and privilege ring 3 being the least privileged user mode where application code runs. Figure 1.1 shows this concept and we will briefly discuss how it is applied to different architectures in the coming chapters.

As applications became more complex, specifically with the advent of largescale virtualization and the internet, this simple security model broke down as executed code could no longer be trusted, nor its origin verified. The problem of "secure remote computation" arises where the data owner must trust not only the software provider, but also the remote computer and infrastructure on which that software is executed. Homomorphic encryption solves the problem of secure remote computation to some extent, however the performance overhead of this transaction limits its application [22].

In an attempt to address the problem of secure remote computation, microprocessor designers have implemented different types of Trusted Execution Environments (TEEs), first defined by the Open Mobile Terminal Platform and ratified in 2009 [45]. These TEEs are intended to allow for code and data to reside in a specially provisioned area of memory where the processor can reserve access rights using a given set of rules. The processor will then guarantee the confidentiality 


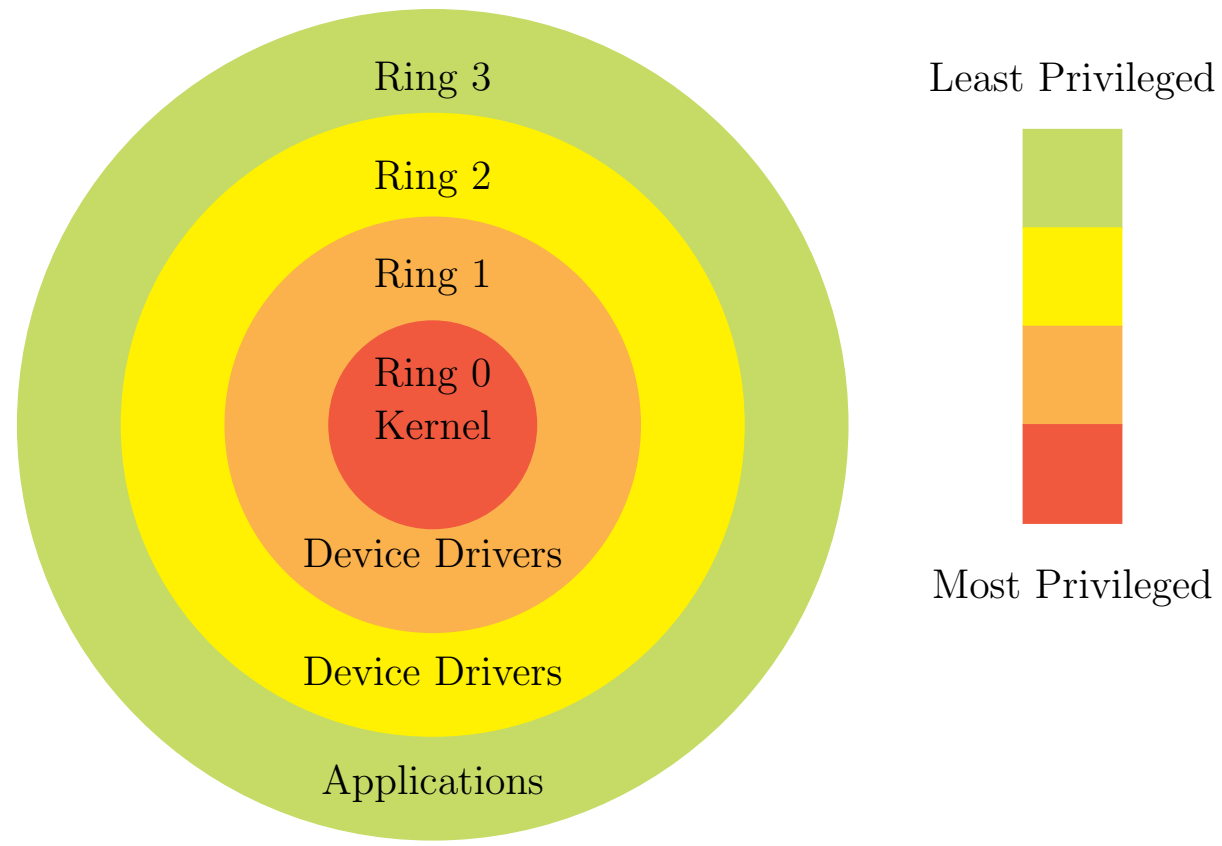

Figure 1.1: Hierarchical protection domains or protection rings. Processors will define the method for switching between modes in such a way that code running in "ring 3" should not have access to code or data running in the rings below it. Attempts to access memory or to run special privileged instructions should throw an exception. Device drivers may run at a higher privilege level to gain access to special instructions. Figure recreated from Hertzsprung at English Wikipedia, CC BY-SA 3.0, via Wikimedia Commons.

and integrity of that region of memory based on the configuration of the system.

In choosing a TEE, hardware architects have many complex features to consider before picking a platform. Currently, many resources exist that cover the features and design details of specific TEEs, however there are few resources that combine this information into one source for comparison. In this thesis, we will examine Intel Software Guard Extensions (SGX), Arm TrustZone, and RISC-V Physical Memory Protection (PMP) in order to provide a methodology for comparing and evaluating TEEs by considering these technologies' respective strengths and weaknesses. The goal of this thesis is not to characterize one technology as overall superior to another, nor is the goal to expose fatal flaws that make one technology 
inherently insecure. Rather, we will describe the properties of a hardware TEE and illustrate how each technology implements those properties. A comparison of these different implementation details yields a method of evaluating TEEs for those looking to choose the TEE that best fits their needs.

These three technologies were chosen for specific reasons. Intel SGX is available on most consumer desktop/laptop products, and the technology is now becoming popular on their Xeon line of server/workstation processors. Arm is the de facto architecture used in cell phones and embedded devices. The popularity of these two architectures makes them a natural choice for comparison. The third technology, RISC-V PMP, is a newcomer to the stage of TEE options. We will examine how the more mature technologies have informed choices made by the architects of RISC$\mathrm{V}$, as they are clearly addressing specific limitations with current technologies. While the method used to examine these technologies will be restricted to those aforementioned three TEEs, this method can be applied to any TEE.

The goal of this thesis is to provide hardware implementers with a method for comparative analysis and not to act as a definitive guide to any one of these three technologies. Any hardware implementation details are given only as examples and are not intended to be used in production systems. Furthermore, this thesis is an examination of the hardware and not the many possible software implementations available. As such, while we may discuss firmware and software throughout the thesis, it is not meant to be a guide to properly implementing a complete solution.

This thesis will begin by giving a brief history of TEEs as well as some of the cryptographic underpinnings of the technology (chapter 2). In considering these topics, we will provide definitions for many of the technologies used in a TEE in order to constrain ourselves to hardware implementations only. We will then 
give a detailed analysis of Intel SGX (chapter 3), Arm TrustZone (chapter 4), and RISC-V PMP (chapter 5). We will examine how these technologies provide the fundamental properties of a hardware TEE: code integrity, data integrity, and data confidentiality. Furthermore, we will describe how some of these solutions can provide more advanced features like code confidentiality, authenticated launch, programmability, attestation, and recoverability. Lastly we will provide a comparison of the three technologies and their methods for providing the fundamental and advanced properties of a hardware TEE (chapter 6). 


\section{Chapter 2}

\section{Background}

\section{$2.1 \quad$ Overview}

In order to understand Trusted Execution Environments (TEEs) we must first look at how computer architecture security worked before the problem of secure remote computation. The processor modes discussed in the introduction contained four privilege rings with the most privileged level, privilege ring 0, executing operating system kernel code. However, in most modern x86 client systems there are 3 levels of even greater privilege than privilege ring 0 . We will examine each of these briefly and describe why they do not provide the same type of protections that a TEE provides.

\subsection{The Predecessors of the TEE}

In 1990, Intel integrated a new privilege mode into their i386SL processor called System Management Mode (SMM) [67], now commonly referred to as "privilege ring $-2 "$ (privilege ring negative two). OS code does not have access to this privilege level and only specific firmware, usually provided by the platform manufacturer, is allowed to execute code in SMM. This processor mode is entered by using a special System Management Interrupt (SMI). This interrupt has the highest priority of any interrupt as of the writing of this thesis, and is even higher priority than a non-maskable interrupt (NMI). Common tasks one might perform in SMM include thermal management, power management, or even something as simple as altering the volume output in response to buttons on a laptop keyboard [66]. SMM 
would remain the most privileged level of code execution until the release of Intel Management Engine (Intel ME) in 2008 [19].

The memory used in SMM, called System Management RAM (SMRAM), is secured from any accesses or modifications originating anywhere outside SMM. Secured accesses include any accesses or modifications originating from the CPU, from I/O devices, and from Direct Memory Accesses (DMAs). When an SMI is generated, all the CPU threads enter SMM and each of their register states are saved in a memory table inside SMRAM. Work can now be done by the SMI handler. Once this work is finished, each of the CPUs previous states are restored and execution is handed back to where the SMI was originally generated. Since there can be multiple SMI handlers there is firmware code that runs in a single thread and acts as an SMI dispatcher. All other CPU threads wait inside SMM until the handler thread has returned [18]. This firmware code is an implementation of the Unified Extensible Firmware Interface (UEFI) specification, which is the successor to the Extensible Firmware Interface (EFI) specification [68].

There are several reasons why SMM is not a suitable replacement for a TEE. Firstly, using SMM for any tasks which consume a significant amount of time would cause the system to hang while SMIs are handled. As such, SMM tasks are confined to small workloads which can return quickly and do not need to happen in rapid succession. However, tasks which take significant amounts of time and may occur in rapid succession are exactly the types of tasks we wish to run in a TEE. Tasks like processing a credit card payment, or verifying the identity of the user with biometrics would all take significant time to run and would need to happen in quick succession.

Secondly, SMM is very restrictive in terms of how code is developed for the 
platform. All code executed in SMM must be located inside the system firmware, loaded into SMRAM, and locked before the OS is loaded into memory. As such, firmware code does not have easy access to communicating with the host OS but must use special EFI SMM variables [60].

Lastly, while updating firmware in the field is possible [69], it almost always requires a reboot of the system. Updating the code in a TEE is a critical function that will be required much more often than a firmware update and should not require system reboot each time. These are just a few reasons why SMM is not a suitable solution to the problem of secure remote computation. Perhaps the overarching reason is that SMM was not designed to solve the problem of secure remote computation. Regardless, we have shown why it should not be used where a TEE would be better suited.

In 2005 [61], Intel released their first processor to allow the next most privileged mode of their x86 processors, Intel Virtualization Technology (VT-x) ${ }^{1}$. Commonly referred to as "privilege ring -1" (ring negative one), VT-x added new instructions called Virtual Machine Extensions (VMSs). These instructions are used to create a layer of hardware isolation between the host OS from the guest OS. It is tempting to see a TEE as a type of virtualization. Indeed, one early form of a trusted computing environment used a "dedicated closed virtual machine" [21] to achieve many of the goals of a modern TEE. However, as others have shown [54], this type of virtualization fails to cover several key properties of a TEE. For our purposes, we will consider these properties attestability and code confidentiality, and will discuss these in depth in coming chapters.

\footnotetext{
${ }^{1}$ We use the acronym VT-x here to refer to all of Intel Virtualization Technologies, though in this case the " $\mathrm{x}$ " refers to Xeon processors with this feature. Non-Xeon processors would use the designation VT. There is also Intel Virtualization Technology for Directed I/O (VT-d). In this thesis we will use VT-x so as to avoid any confusion with the common acronym VT.
} 
In 2008 [19], Intel developed what is today the most privileged processor level on x86 hardware, the Intel Management Engine (Intel ME). This technology, commonly referred to as "privilege ring -3" (privilege ring negative three) is not actually a processor mode at all. It is instead a feature of some Intel chipsets. Most of the properties of Intel ME are obscured by the proprietary firmware that it runs. The majority of information available regarding Intel ME is found either in Intel's publications or from the plethora of reverse engineering done by security researchers. As such, we will not consider Intel ME a suitable replacement for a TEE for two key reasons: most of the information about this technology is gained by indirect means like reverse engineering and the firmware is not intended to be modified from the signed binary provided by Intel.

These three x86 "negative privilege rings" provide hardware protection of various kinds and each has its own restricted area of memory. However, none of these technologies serve as a viable solution to the problem of secure remote computation. It is no surprise that Intel eventually developed Software Guard Extensions (SGX), their own implementation of a TEE in order to address this problem. Before we address SGX specifically, we will continue to track the development of Trusted Execution Environments (TEEs) by starting with their use in handsets, the precursor to today's smartphones.

\subsection{Birth of a TEE}

The concept of securing computation is not a new idea. As we have seen with Intel ME, neither is the technique of using a processor other than the main, general purpose CPU for secure computation. For almost two decades [47] hardware manufacturers have relied on Trusted Platform Modules (TPMs) for a similar kind 
of secure computation. In 2009 the Trusted Computing Group (TCG) specification for a TPM was ratified as an ISO standard. A TPM is a system that must remain separate from the system it reports to: the host system. It can be a single physical component that communicates with the host system over a simple bus. TPMs can have their own processor, RAM, ROM, and flash memory. While the host system cannot directly change the values of the TPM memory, it can use the simple bus to send and receive information. As of TPM version 2.0, the TPM can now be a part of the main processor, however it must use hardware memory partitioning and use a well defined interface instead of the simple bus. The use of the main processor as a TPM may add significantly more speed, but at the cost of more complexity. TPMs contain random number generators, cryptography key generators, and secure storage.

While TPMs have many of the features we require of our TEEs, they were designed with securing small amounts of data like cryptographic keys, not entire applications. TPMs focus on security over speed and their cryptographic algorithms may be purposely run at much slower speeds than is possible with a CPU, let alone a cryptographic accelerator. TPMs do, however, provide a valuable tool for a TEE to use as part of its chain of trust ${ }^{2}$. Concepts used in a TPM like secure storage and isolated memory are expanded in a TEE with concepts like isolated and protected IO and isolated RAM. It is not surprising that we see the first industry efforts around TEEs around the same time that the ISO standard for TPMs is published.

\footnotetext{
${ }^{2}$ Unsurprisingly, the concept of a "chain of trust" comes out of electronic commerce security publications from the 1990's [40]. The internet opened up the concept of electronic commerce to a much broader audience. In doing so, it also opened up a much larger attack surface for threat models to consider.
} 
TEEs were first defined by the Open Mobile Terminal Platform (OMTP) Hardware Working Group and ratified in 2009 [45]. This standard was designed specifically for "handset manufacturers". The OMTP standard was transferred to the Wholesale Applications Community (WAC) in 2010 and in July 2012 WAC itself was closed, with the OMTP standards being transferred to The GSM Association (originally Groupe Spécial Mobile) [48]. The OMTP standard defines an "execution environment" as a combination of five elements: a processing unit, a bus, physical memory, a boot process, and the contents of the execution environment [45]. These contents include the code, data, and keys required for computation. The OMTP document goes on to describe two sets of security requirements which meet their definition of a TEE, called "Profile 1 and 2". Profile 2 provides greater security than Profile 1, however both meet their definition of a TEE.

Both profiles provide protection against unauthorized memory access given a list of vulnerable attack surfaces summarized in Table 2.1. The core requirements for an OMTP TEE cover these "threat groups" to different degrees. There are 27 core requirements in total and it is out of the scope of this thesis to list or go into details on them. Sufficed to say that they address the attack surfaces described in Table 2.1. Also, it is clear from the threat groups described that these threat models are only applicable to handsets and do not cover typical embedded devices. Another standards body called GlobalPlatform took up the task of standardizing the use of TEEs across multiple embedded platforms.

GlobalPlatform Incorporated is a nonprofit industry organization that began in 1999 with the mission of providing standardization around mobile payment devices and software. In 2011 they created a TEE model [25] that met the needs of embedded use cases. They define a TEE as a system providing "isolated execution, 


\begin{tabular}{|l|l|}
\hline Group & Attack Surface \\
\hline Group 1 & $\begin{array}{l}\text { Hardware modules used for accessing memories, only including at- } \\
\text { tacks mounted via modules built into the "mobile equipment" de- } \\
\text { sign (e.g. DMA module) }\end{array}$ \\
\hline Group 2 & $\begin{array}{l}\text { Colour LCD controllers or "graphics chip of the mobile device" } \\
\text { designed to be pointed at memory blocks and could dump that } \\
\text { information to the screen }\end{array}$ \\
\hline Group 3 & Removal of battery or external memory card \\
\hline Group 4 & Replacement of flash when power is off \\
\hline Group 5 & Extract secrets by bus monitoring (e.g. hardware probes) \\
\hline Group 6 & $\begin{array}{l}\text { Attached devices (mod chips) used to attack data between flash } \\
\text { and the System on Chip (SoC) or in external RAM. }\end{array}$ \\
\hline Group 7 & Replacement of flash when power is on \\
\hline
\end{tabular}

Table 2.1: Attack surfaces as grouped by the OMTP. This table is created from data available in section 2.1 of the OMTP document. [45]

integrity of Trusted Applications (TAs), and integrity and confidentiality of TA assets" [25]. A GlobalPlatform TEE is more rigorous than the OMTP standard in that it requires a security certification provided by GlobalPlatform and must comply with the following GlobalPlatform standards: TEE Protection Profile, TEE Client API Specification, and TEE Internal Core API Specification [23, 24, 27].

Unlike in the OMTP standard, GlobalPlatform first introduces the concept of attestation in its definition of a Root of Trust (RoT). We will discuss attestation in depth for each of our technologies we cover, as well as in chapter 6 where we consider this property of a TEE and if it can be considered optional [58]. Standards 
and specifications from groups like OMTP and GlobalPlatforms paved the way for the creation of mobile devices capable of secure remote computation. Indeed, the smartphone revolution would only have been possible with the ability to run trusted applications allowing users to interact with financial, healthcare, and other valuable data.

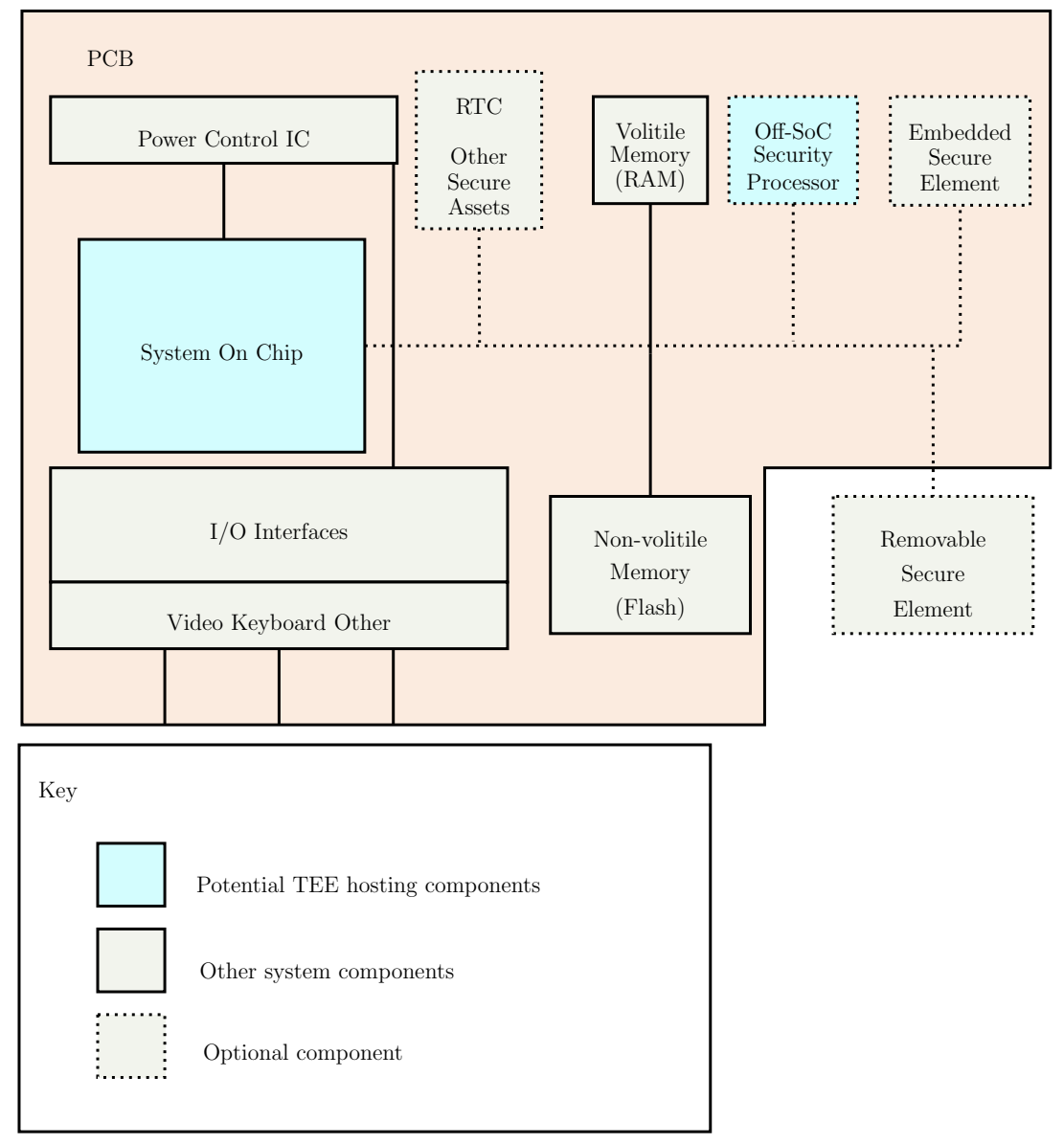

Figure 2.1: The board level chipset architecture of a typical mobile device. The concept of an embedded or removable secure element allows for devices like a TPM which may hold secure data but will not host the TEE. A TEE may reside on the SoC itself as with Arm TrustZone. However, it is possible to physically separate the TEE from the SoC and still be compliant with the GlobalPlatform model. This figure was recreated and simplified from Figure 2-1 on page 15 of the GlobalPlatform Technology TEE System Architecture Version 1.2. [25].

In Figure 2.1 we see a very common mobile device system diagram with the 
possible TEE hosting components listed as both on and off chip. GlobalPlatforms differentiates between the Rich Execution Environment (REE) and the Trusted Execution Environment (TEE). The REE focuses on extensibility and versatility whereas the TEE has only one purpose: to "provide a safe area of the device to protect assets and execute trusted code" [25]. These standards were first put into use by complex SoCs with the release of processors implementing the ARM Cortex A5 core in 2012. These cores included Arm TrustZone as well as a reference firmware implementation for building secure applications. Arm TrustZone has since become the de facto standard for embedded devices as well as as Androidbased mobile phones requiring a TEE.

Up to now, we have only covered relatively simple use cases like handsets, smartphones, and embedded devices. Bridging the gap between these simple devices and desktop or server processors will require we cover the current dominant architecture in that space, namely the x86 family implemented by Intel and AMD.

\subsection{From Handsets to the Cloud}

In 2013 at a workshop titled Hardware and Architectural Support for Security and Privacy (HASP), Intel introduced Software Guard Extensions (SGX) for Intel architecture. SGX is not an extension to the $\mathrm{x} 86$ architecture, but rather to the Intel architecture. As such, AMD developed their own solution called Secure Encrypted Virtualization (SEV). As mentioned previously, we will not discuss AMD SEV in this thesis as its architecture is quite different from Intel SGX. While there are sev-

eral papers published in the proceedings from the HASP 2013 workshop [1, 30, 42], as well as two patents from Intel's original filings $[32,41]$, we will use a more recent paper by Costan [15] as well as a recent whitepaper from Intel [31] in exploring 
SGX.

Intel's introduction of SGX opened up the desktop and laptop markets to a new type of software application capable of hardware-backed secure "enclaves". It also gave application developers on Intel platforms the chance to utilize both local and remote attestation, providing assurance from the vendor regarding code integrity and confidentiality. It wasn't until 2021 that Intel would make this technology available in multi-socket Xeon CPUs, opening the door for cloud providers to take advantage of TEEs on Intel platforms [13].

\subsection{RISC-V: An Open Source TEE}

RISC-V is a popular modern architecture developed at the Parallel Computing Lab (Par Lab), which is part of the University of California at Berkeley. Perhaps the most notable thing about this architecture is that it has been developed using the same methodology as open source software. The specifications are published using a Creative Commons license, and all code examples or artifacts of the specification use permissive licenses like the BSD licenses or the Apache license [62]. The work done on the RISC-V specifications is overseen by a non-profit organization called RISC-V International, an organization registered in Switzerland.

The Trusted Execution Environment Task Group of RISC-V International has developed a specification called Physical Memory Protection (PMP) as part of the RISC-V Privileged Specification [63]. RISC-V PMP extends the concept of Physical Memory Attributes (PMA) to include multiple configurable sections of memory whose access rights can be altered by firmware running at the highest privilege level. RISC-V PMP is certainly a very basic type of TEE, however plans to 
extend its capabilities have already been drafted in a new specification called Extended Physical Memory Protection (ePMP). Likewise, drafts of S-Mode Physical Memory Protection (sPMP) and I/O Physical Memory Protection (IOPMP) are set for ratification in 2021. We will discuss all of these types of memory protection in detail in chapter 5 .

This chapter has given a brief background of Trusted Execution Environments (TEEs) and we have been selective in mentioning only a few of the many examples of hardware security that influenced the development of TEEs. We have shown that any given TEE builds off the technologies of the past, even when incorporating new instructions or hardware unique to TEEs. As we will see, without technologies and standards like the TPM ISO standard of 2009, TEEs would not have the means to perform many of the advanced features like attestation which are critical to some applications. We continue now with an overview of our three technologies for comparison: Intel Software Guard Extensions (SGX), Arm TrustZone, and RISC-V Physical Memory Protection (PMP). 
A Timeline of TEE Related Events

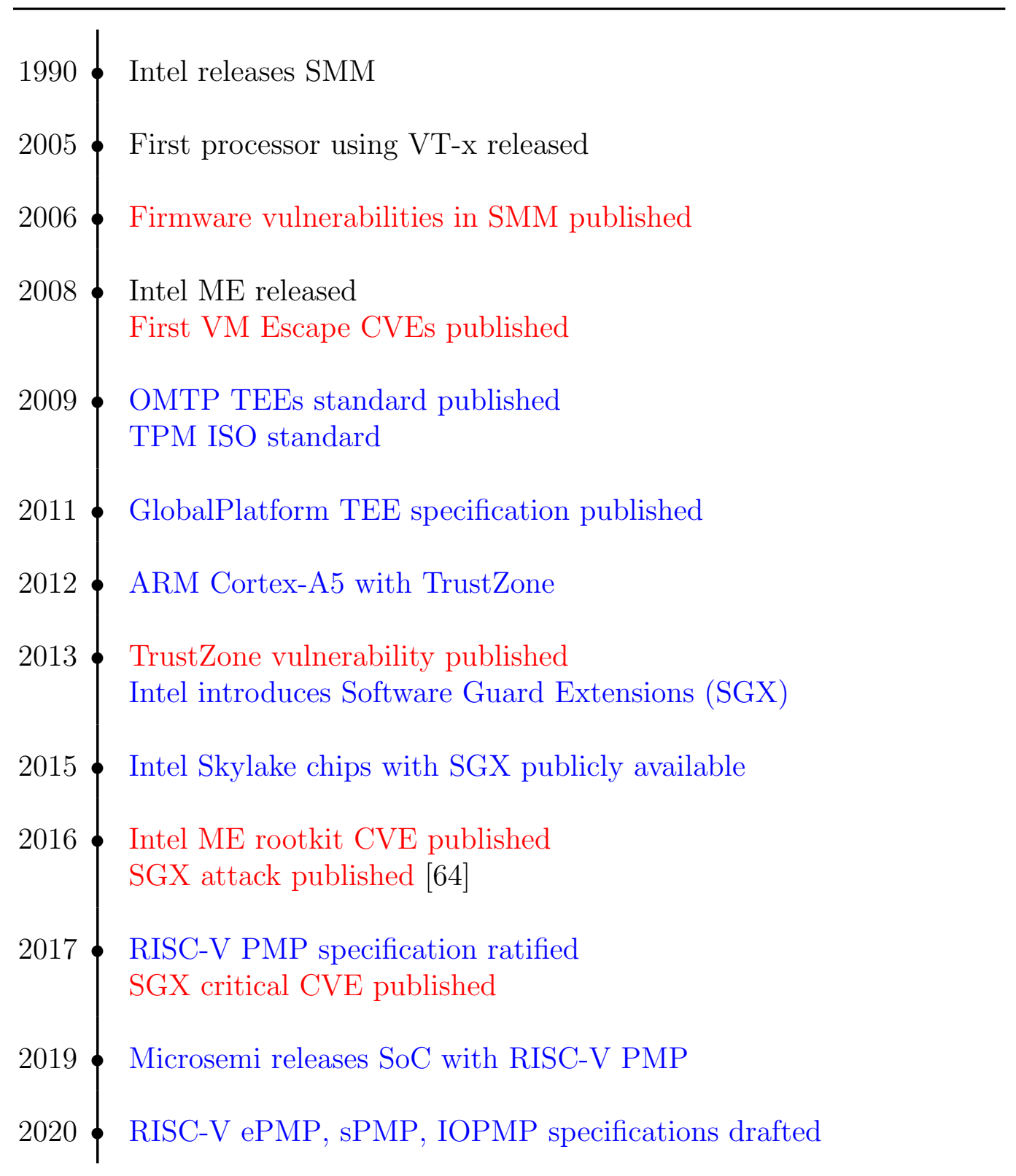

Figure 2.2: An overview of modern hardware security features, specifications, and vulnerabilities In this timeline, events pertaining to TEEs are in blue and vulnerabilities in hardware security technologies are in red. Dates of vulnerabilities are not exact, see https://cve.mitre.org/ for exact dates and severity. Dates of technology releases are estimates and taken by the first broadly available product release with the given feature available. 


\section{Chapter 3}

\section{Intel Software Guard Extensions}

\subsection{The Intel SGX Solution}

Intel Software Guard Extensions (SGX) is built on designs of software attestation already proven in technologies like the Trusted Platform Module (TPM) and Intel Trusted Execution Technology (TXT). In SGX, these concepts of software attestation are used to create containerized sections of memory on the remote computer called "secure enclaves" where data and code can be loaded or executed securely. These enclaves are verified by both a cryptographic attestation key of the container's contents as well as a hardware Root of Trust (RoT) manufacturer's key. Unlike the TPM and TXT technologies, SGX securely operates only on a small amount of data and code called the Trusted Compute Base (TCB), leaving the majority of memory outside this TCB.

\subsection{Initial SGX Enclave Setup}

Configuration settings for SGX exists as part of the platform firmware, and most firmware vendors provide simple tools for enabling SGX. If SGX is enabled, the firmware is responsible for setting aside a memory region called the Processor Reserved Memory (PRM), and most firmware tools allow specifying the size of the space allocated. The firmware allocates the PRM by setting a pair of model-specific registers (MSRs), collectively known as the PRMRR. The CPU will then protect the PRM from all non-enclave memory accesses including kernel, hypervisor and System Management Mode (SMM) accesses, as well as Direct Memory Access 
(DMA) from peripherals [15].

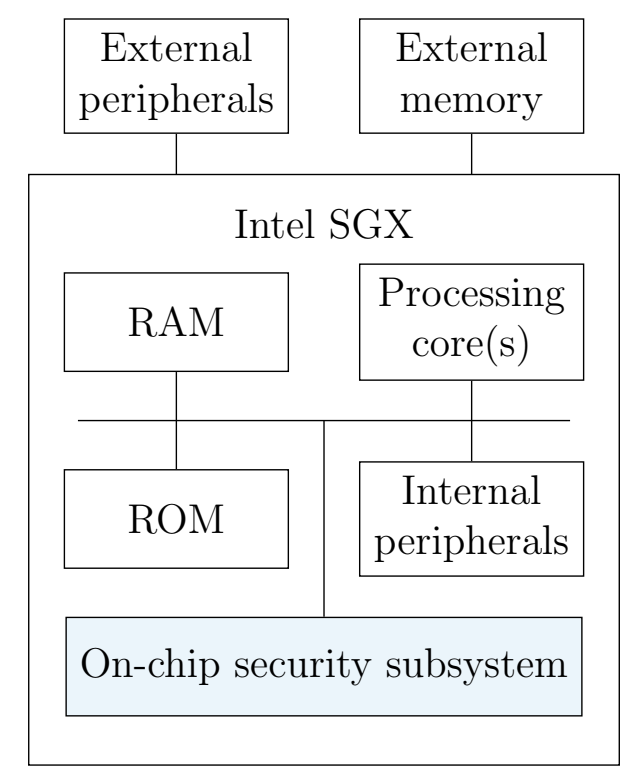

\section{Typical Intel SGX Processor}

Figure 3.1: A high level overview of Intel SGX layout on a processor. Both the processor and the platform firmware, which on Intel systems will likely be an implementation of Unified Extensible Firmware Interface (UEFI), must support SGX. The rest of the platform, including external peripherals and memory, have no knowledge of SGX. The details of how Intel implements SGX within the processor are not documented in detail publicly. Reproduced as a simplified version of Figure 2-3 of the GlobalPlatform specification [25].

This section of specially allocated memory is used to store the Enclave Page Cache (EPC), which are the 4kb pages holding both the enclave data and code. The exact layout of the PRM and EPC are model-specific, and depend on firmware settings. While untrusted system software both assigns these EPCs to an enclave and loads them with data, it is the CPU which keeps track of all the EPCs ensuring that they only belong to one enclave. Once the system software loads data into the enclave it asks the CPU to mark that enclave as initialized, after which no 
other data may be loaded into the enclave as this setup process is disabled for that enclave. After initialization, a measurement of the enclave is taken by means of a cryptographic hash. Checking that measurement later ensures that any operations performed on the enclave are done so in a secure environment.

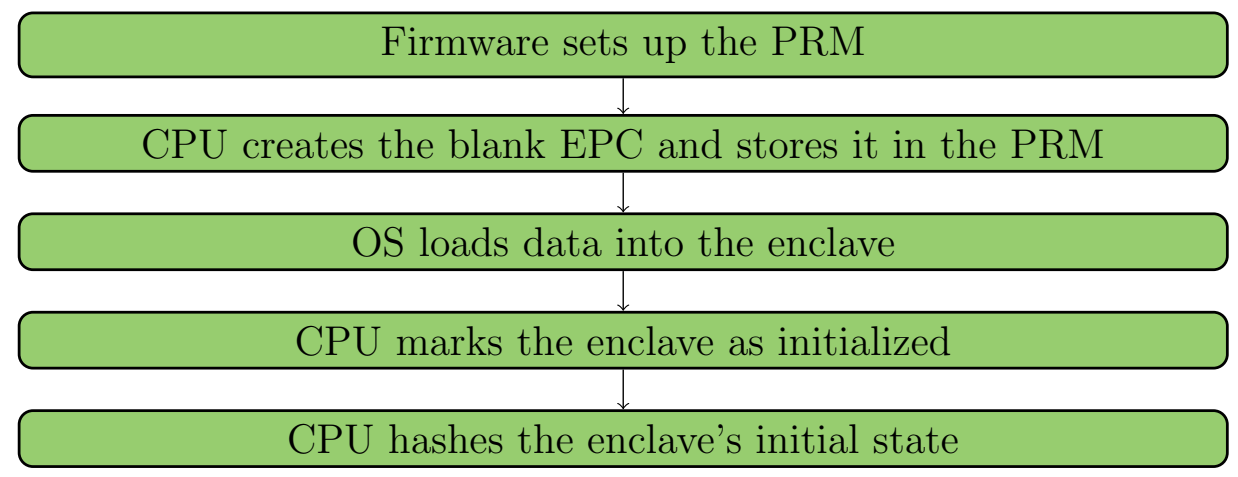

Figure 3.2: Workflow for setting up an enclave. It is important to note that the firmware which sets up the PRM and creates the EPC is vendor specific. As of the writing of this thesis, it is highly probable that the firmware is based on the open source project TianoCore, an implementation of UEFI spearheaded by Intel in 2006 and now led by engineers from companies like Intel, Arm, Apple, and Red Hat.

\subsection{Executing SGX Enclave Code}

Execution flow can only move into an enclave via a special CPU instruction EENTER, much like switching from user mode to kernel mode. The actual execution happens in "user mode" also known as "privilege ring 3" and takes advantage of address translation from the operating system or hypervisor. The benefit to this model is that the OS or hypervisor will quickly provide address translation lowering overhead. As Costan notes, "the operating system and hypervisor are still in full control of the page tables and extended page tables (EPT), and each enclave's code uses the same address translation process and page tables as its host application. This minimizes the amount of changes required to add SGX support 
to existing system software." [15]. The downside is that code executing inside the enclave does not have access to system calls (syscall) or any other high privilege operations. An inability to make system calls limits the types of operations that can be preformed inside an enclave. The code executing inside the enclave does have access to its entire address space which includes the "host application" that caused the creation of the enclave.

The CPU executing the enclave code will perform an Asynchronous Enclave Exit (AEX) whenever execution moves outside the enclave such as servicing an interrupt or during a page fault. The CPU state is saved inside the enclave memory metadata before exiting, ensuring that the CPU can securely restore the state of enclave execution. There are special machine mode CPU instructions that are used both in allocating EPC pages to the enclave as well as evicting those pages into untrusted DRAM. Page management instructions allow code outside the enclave to operate on code within the enclave. SGX uses cryptographic protections to assure the confidentiality, integrity, and "freshness" [15] of the evicted EPC pages while they are stored in untrusted memory.

As mentioned the application that makes calls into an enclave or "host application" lives in the same address space as the enclave code and data. Restricting host and enclave to the same address space has benefits in terms of application size variability, but may open the host application up to attack should the enclave code become compromised [55]. SGX allows enclaves to execute multiple threads through a Thread Control Structure (TCS) which allows multiple logical cores to execute enclave code. Within the EPC metadata, reserved secure memory called the State Save Area (SSA) allows the threads to save their state when a context switch happens, like servicing an interrupt. In this way, Intel SGX is able to allow 
a specific amount of code and data to remain protected while still allowing access to that data by code outside the trust boundary.

\subsection{Life Cycle of an SGX Enclave}

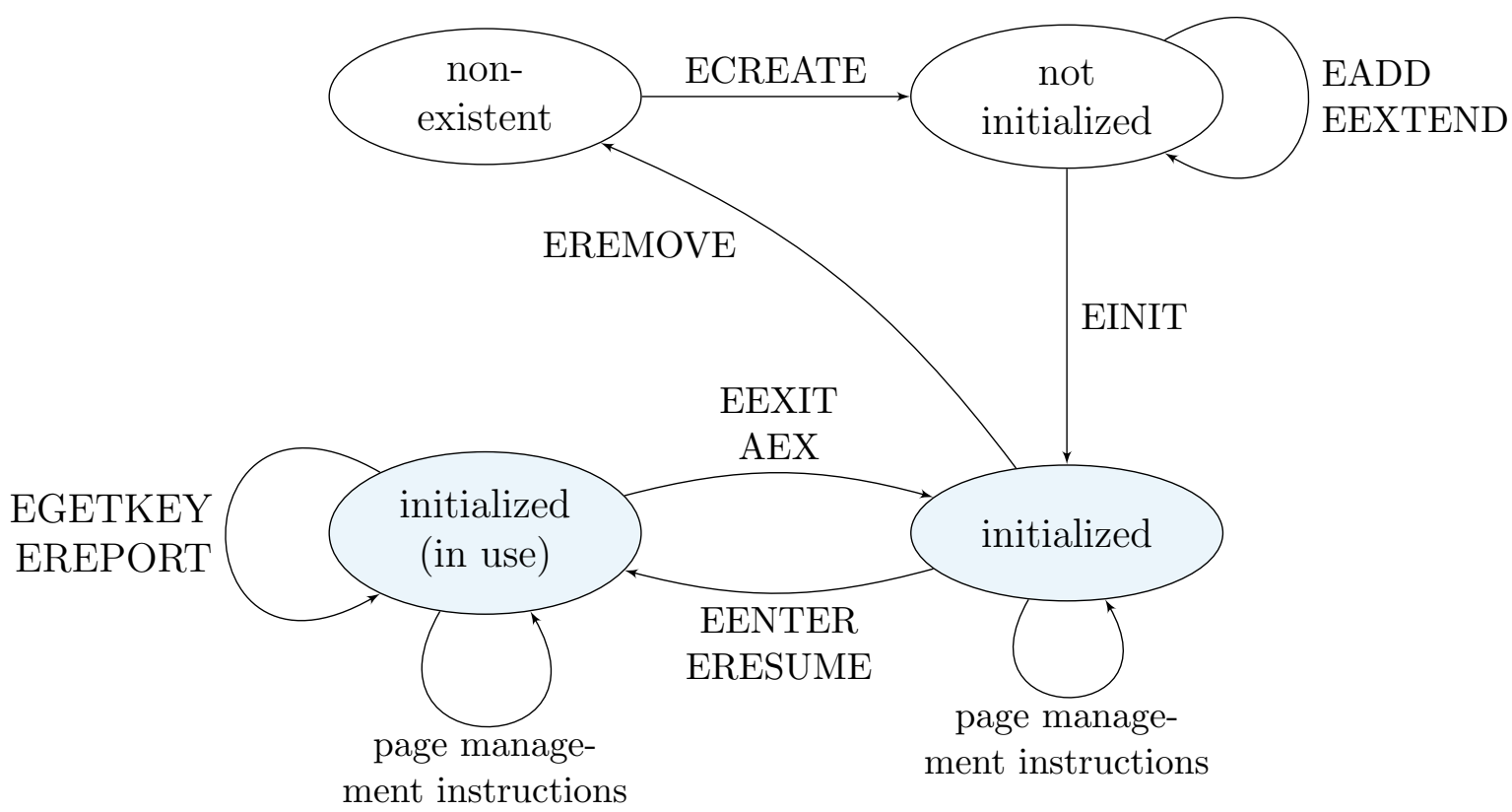

Figure 3.3: Intel SGX enclave life cycle. The enclave's memory is protected in states shaded blue. Note that in the enclave is not secure while in the uninitialized state as pages are being added or extended. Until a measurement is loaded into the MRENCLAVE register, code and data integrity can not be assured. The CPU will call AEX in order to service interrupts, and the state of the enclave will be saved in the SSA. Reprinted as a simplified version from Costan's Figure 63 [15].

In order to understand the life cycle of an enclave, we must consider the specific x86 instructions used to create and manage these enclaves. Many of these instructions which create enclaves, extend pages, and remove enclaves operate in "privilege ring 0", one of the most privileged modes. Whereas attestation, entering, and exiting the enclave can be done from "privilege ring 3 " the least privileged 
mode. The first of the privileged instructions in our life cycle is ECREATE which fills a protected data structure located inside the EPC with the size and measurement hash of the enclave. This data structure, called the SGX Enclave Control Structure (SECS), is used by the hardware and is not directly accessible to software. The system will then add pages to the EPC with the EADD instruction, and extend the EPC page measurement with the EEXTEND instruction. When executing EADD and EEXTEND the system will ensure that the page's address range is within the Enclave Linear Range (ELRANGE). The EEXTEND instruction allows for the accumulation of a hash of all the pages in the EPC. This measurement can be used later for attestation that the enclave has not been tampered with or changed in some way.

It is important to note that in its uninitialized state, none of the enclave code or data is encrypted. For example, any privileged code running at "privilege ring 0" can gain access to these data and structures. Enclaves must be initially built on a system that is known to be secure, such that the measurements taken are considered a "gold standard" with which to preform attestation on a local or remote machine at some later time. When the EINIT instruction is called, the enclave is considered fully built, the measurement is locked down, and "privilege ring 3" (user) applications can now enter the enclave and attest that it contains the code and data that the developer intended.

EBLOCK, ETRACK, EWB, and ELOAD ${ }^{1}$ are paging instructions run with "privilege ring 0" privileges. The goal is to allow the paging of secure pages into and out of main memory while ensuring the confidentiality and integrity of those

\footnotetext{
${ }^{1}$ Actually, two load commands, ELDB and ELDU both load into memory a previously evicted page, with ELDB for blocked and ELDU for unblocked. Pages may be blocked when being prepared for eviction. All future accesses to blocked pages will result in a page fault.
} 
pages. Information stored inside the EPC called the Paging Crypto MetaData (PCMD) keeps track of the identity of the enclave the page belongs to and a pointer to an access rights structure. There is also a Version Array (VA) which is used to store the version numbers of pages evicted from the EPC. These versioned and access controlled pages are therefore hardware protected, and any change to the versioning, access rights, or origins of the page will result in a page fault. It is possible to have 2 instances of the same enclave, however pages cannot be swapped between them, and the hashes of these pages will not be the same.

Once an application has requested that "privilege ring 0" components build the enclave and EENTER is called, the enclave may begin execution. The hardware is responsible for saving (AEX) and restoring (ERESUME) the architectural state of execution should any external events like interrupts or exceptions cause execution to leave the enclave. The EGETKEY and EREPORT instructions operate in user mode ("privilege ring 3") and seal data based on the key the developer provides. Using these two instructions SGX applications operating in "privilege ring 3" are

able to preform local attestation of the enclave, perhaps the most vital function of any Trusted Execution Environment (TEE). Finally, once the enclave is no longer needed, the system will call EEXIT, and a synchronous enclave exit will occur. As Costan notes, "EEXIT does not modify most registers, so enclave authors must make sure to clear any secrets stored in the processor's registers before returning control to the host process." [15].

\subsection{Attestation with Intel SGX}

Software attestation of enclaves is required to ensure the integrity of the enclave. This attestation can happen locally between two enclaves on the same platform or 
remotely between two different platforms. As previously noted, the measurement of the enclave includes a SHA-256 hash of the enclave's attributes as well as the content, position, and access rights of its pages. This measurement is stored in a register called MRENCLAVE which represents the enclave's TCB. The EREPORT instruction is used to generate a signed report of this TCB and the EGETKEY instruction then retrieves the key used to validate said report. Local attestation of enclaves can be done using symmetric encryption as the hardware can ensure the integrity of the single key being used to verify the MRENCLAVE value. Remote attestation must be done using asymmetric encryption (both a public and private key) and requires the remote SGX enabled platform to query an Intel attestation server.

Local attestation can be used by enclaves running on the same platform and will provide assurance as to the identity of the enclaves. The local enclave which attests to the reporting enclave is called the "Quoting Enclave" [31]. Figure 3.4 provides a high level overview of the attestation process including both local and remote attestation. On today's complex and connected systems, local attestation alone is not enough to assure that the platform and enclave are genuine SGX enclaves [34]. Remote attestation requires asymmetric keys as well as a remote server with which to verify the quote of an enclave.

Intel platforms include two statistically unique "root keys" which provide assurance that the system is indeed a genuine Intel SGX platform. These include the Root Provisioning Key and the Root Seal Key [31] which are stored in hardware fuses. Intel also provides a more advanced form of asymmetric encryption called Enhanced Privacy ID (EPID). EPID is essentially a group signature where the system can attest to a message being signed by a member of the group without 


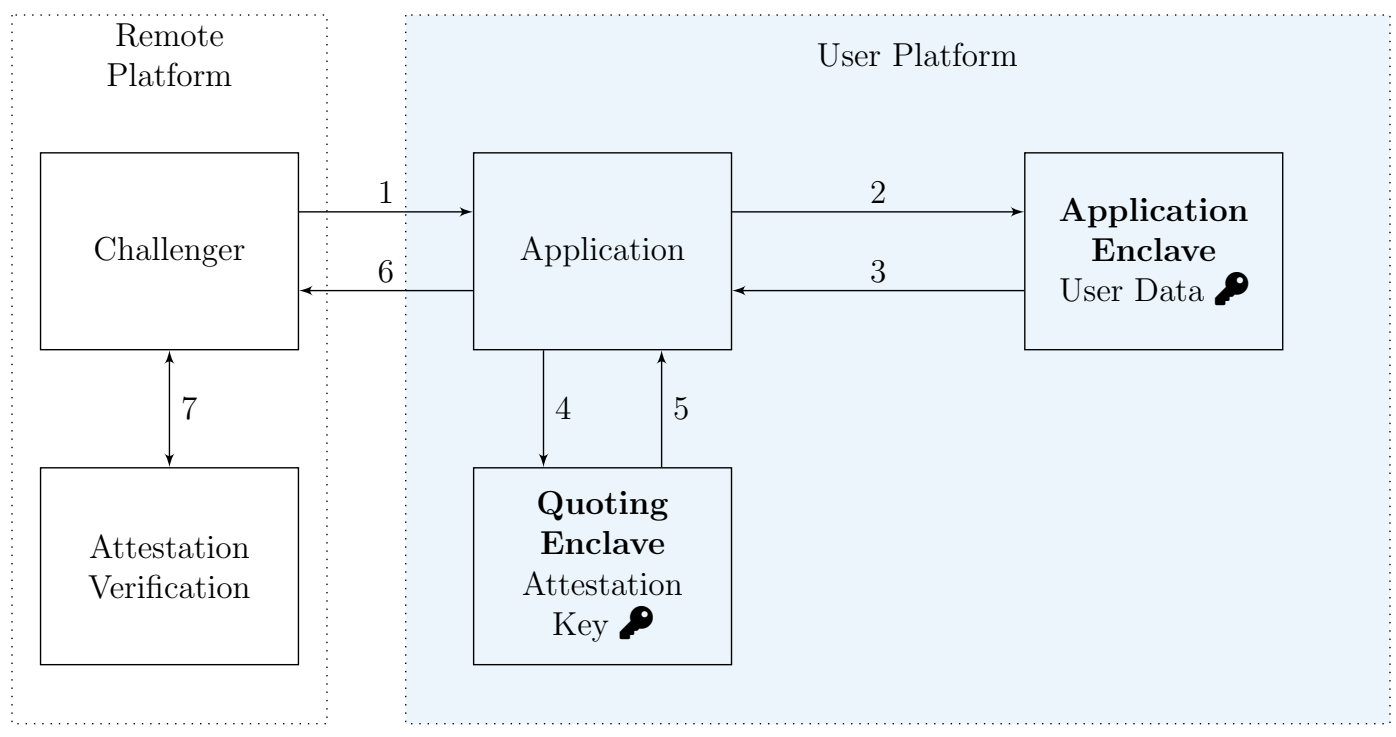

Figure 3.4: Local and remote attestation of an Intel SGX enclave. A remote challenger may request that an application provide attestation. The application must then request a report from the enclave and locally verify that against a quoting enclave. The quoting enclave will use the remote along with asymmetric keys to produce a remote attestation "quote" to be returned to the challenger. The challenger may then use some verification service to check the validity of the quote. This figure was reproduced from Figure 1 [31].

divulging the identify of the signer. The signature algorithm is nothing new [11] and has been included in the ISO/IEC 20008 and 20009 standards. EPID includes several revocation schemes which allow keys to be revoked based on checks performed by the verifier and/or issuer. The Intel Attestation Service (IAS) will take submission of quotes provided, verify the validity of the signatures, and verify that they have not been revoked.

In this chapter, we have briefly described Intel Software Guard Extensions (SGX) from the initial setup of the enclave through to the remote attestation of that enclave's contents. This system is by far the most complex of the three we will examine in this thesis. We have only covered the properties that are necessary to 
understand if we are to appropriately apply our method of comparison. Depending on the use case, a much more in depth knowledge of SGX may be required. However, this thesis will show that even with this rudimentary understanding, one can still apply rigorous method to analysing features of a TEE. Next, we will cover Arm's framework for building TEEs called TrustZone, as well as the reference implementations of their firmware: Trusted Firmware-A (TF-A) and Open-source Portable TEE (OP-TEE). 


\section{Chapter 4}

\section{Arm TrustZone}

\subsection{The Arm TrustZone Solution}

Arm released TrustZone in 2004 for their "general purpose compute" cores, and only as recently as 2016 did they extend this technology to their cores designed for microcontrollers [51]. When evaluating how Arm's TrustZone works, we must remember several important distinctions. Firstly, the Arm specifications include several different architectures with several different states. Each Arm architecture and state combination may operate slightly differently in regard to how TrustZone is implemented. This thesis will only consider the ARMv8-A architecture ${ }^{1}$ running in the AArch64 ${ }^{2}$ state. Secondly, because Arm Limited licenses their cores to hardware manufacturers, System on Chips (SoCs) and platforms may choose to implement security in many ways, and with much more flexibility than in Intel platforms. For simplicity's sake, this thesis will only cover firmware solutions for TrustZone implementations provided by Linaro's open source projects Trusted Firmware-A (TF-A) and Open-source Portable TEE (OP-TEE).

Arm SoC processors create a more absolute separation between the concepts of "secure" and "normal or insecure" operation than Intel Software Guard Extensions (SGX). At its highest level this is accomplished using the Secure Configuration

\footnotetext{
${ }^{1}$ Arm Limited produces three ISA profiles [3]: A-Profile for general purpose compute, R-Profile for real-time, and M-Profile for microcontrollers. These profiles can be seen in the architecture version naming scheme, such that ARMv8-A is "version 8" of the ARM instruction set, with a focus on general purpose compute. The cores that Arm Limited licenses to customers are labeled "Cortex", with ARM Cortex-A55 referencing a specific microarchitecture implementation of the ARMv8-A ISA.

${ }^{2}$ AArch64 is the 64-bit state of any ARMv8-A core which is capable of also running in the 32-bit state called AArch32 [3].
} 
Register (SCR) "Non-Secure bit" (NS) with 1 meaning non-secure and 0 meaning secure. This is perhaps the most fundamental element that separates Arm's two security worlds. Digging a bit deeper, this separation of worlds is accomplished using four separate primitives: one on the bus, one on the SoC core, one as part of the memory infrastructure, and finally one as part of the debug infrastructure.

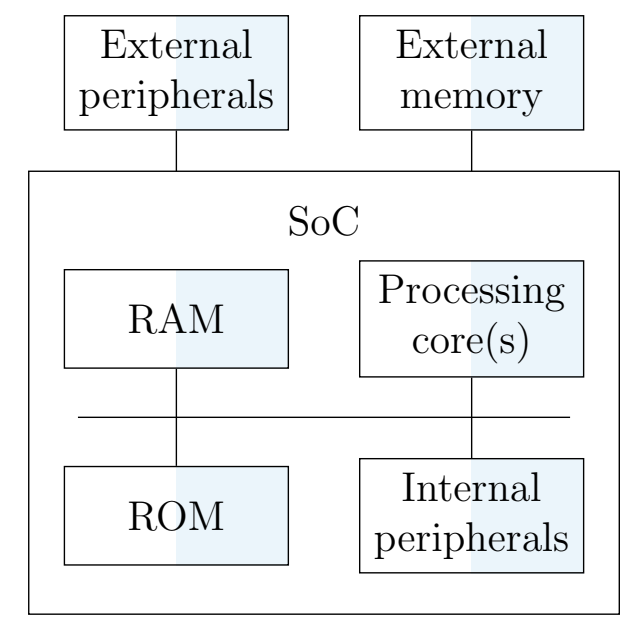

\section{One Possible TrustZone Platform}

Figure 4.1: A high level overview of Arm TrustZone layout on an SoC. Unlike Intel SGX, TrustZone is present as part of much of the SoC's platform. Shaded areas imply that some logic is present which allows TrustZone to secure the Trusted Execution Environment (TEE). Though not shown in this figure, in TrustZone systems, even the bus contains logic separating secure and normal operation. Reproduced as a simplified version of Figure 2-3 of the GlobalPlatform specification [25].

Firstly, the bus interface, called the Advanced Microcontroller Bus Architecture (AMBA) Advanced eXtensible Interface (AXI), partitions all of the SoC's hardware and software resources by taking advantage of a set of bits. Hardware logic present in this "TrustZone-enabled AMBA3 AXI bus fabric" [3] ensures that no "Secure World" resources can be accessed by "Normal World" components. These bits 
include AWPROT for write transactions and ARPROT for read transactions where like the NS bit low is Secure and high is Non-secure.

Secondly, SoCs which implement the ARMv8-A instruction set must also implement extensions which enable a single physical processor core to safely and efficiently execute code from both the Normal World and the Secure World in a time-sliced fashion [44]. The value of the Non-Secure bit is read from the SCR and passed along down the bus to the memory controller and peripherals. A new instruction, the Secure Monitor Call (SMC), is added which allows the core to switch between the secure and normal modes. We will discuss the secure monitor firmware which is responsible for handling these interrupts in the next section.

Thirdly, the memory infrastructure includes security features like TrustZone Address Space Controller (TZASC) and TrustZone Memory Adapter (TZMA) [44]. The TZASC allows for configuration of the secure and normal world memory regions. It does so by partitioning DRAM into areas which have secure world access and those regions which have normal world access. This process can only be done from the secure world. The TZMA serves a similar function for any off-chip memory such as an off-chip ROM. The way in which this memory partitioning happens is based on the specific SoC implementing TrustZone. SoC manufacturers can provide robust or simple partitioning and it is important to understand the implementation of your specific SoC's memory controller to properly understand how TrustZone has been implemented.

Lastly, the security-aware debug infrastructure controls debug access to the Secure World. This includes "secure privileged invasive (JTAG) debug, secure privileged non-invasive (trace) debug, secure user invasive debug, and secure user non-invasive debug" [3]. By using two different signals into the core along with two 
different bits in a secure register, the core can report either invasive or non-invasive debug info. In this way, the core is able to debug either the normal world only, or it can debug both the secure and normal worlds together. These four primitives provide a framework or scaffolding on which to build a platform capable of secure computation.

In the next section we discuss the firmware that will implement a TEE using TrustZone's features. First, we will point out a potentially confusing difference between how Intel and Arm create privilege levels. Unlike Intel platforms which refer to their privilege levels as privilege rings, Arm uses "Exception Levels" EL0 through EL3 [4]. Here EL3 is the highest, most privileged level where as EL0 is the lowest and least privileged level. Much like with the x86 architecture, exceptions like data aborts, prefetch aborts, and other interrupts can be taken from the level at which they occur to the same or any higher privileged level, but not a level which has less privileges. So, for example, an interrupt occurring in the OS kernel (EL1) can be handled in the kernel or in the secure monitor (EL3), but not in the lower privileged application level (EL0). Practically speaking this means that the user applications running on a system which has not been compromised will not have access to kernel or lower exceptions. Another common confusion point between Arm and Intel is that Intel's "privilege ring 0" is the highest privilege level while Arm's "ELO" is the lowest privilege level.

See Table 4.1 for a complete list of privilege levels, their description, and how they might be implemented in an ARMv8-A system. Each exception level manages its own page tables and control registers with the exception of EL0 which is managed by EL1 [4]. This is a common practice across architectures where the kernel level mode controls the page table for the applications running on top of it. 


\begin{tabular}{|l|l|l|}
\hline Privilege Level & Description & Implementation \\
\hline EL-0 & Application Privilege Level & Supported by CPU architecture \\
\hline EL-1 & Kernel Privilege Level & Supported by CPU architecture \\
\hline EL-2 & $\begin{array}{l}\text { Virtualization Privilege Level } \\
\text { (Optional) }\end{array}$ & Supported by CPU architecture \\
\hline EL-3 & Secure Privilege Level & $\begin{array}{l}\text { Supported by CPU architecture } \\
\text { or a dedicated embedded } \\
\text { security processor }\end{array}$ \\
\hline
\end{tabular}

Table 4.1: Arm Privilege Level Mapping

As we will see, this division is taken advantage of by the ARMv8-A architecture to enable the separation of memory accesses between the Secure World and the Normal World.

\subsection{Arm Trusted Firmware}

Since 2013, Arm, in cooperation with several other industry leaders, has provided Trusted Firmware-A (TF-A) as an open source reference implementation of the firmware required to develop Secure World software for A-Class devices (including ARMv8-A). TF-A provides many features including secure device initialization, modular boot flow, trusted boot, and the secure monitor that allows switching between the Normal World and the Secure World. It should be noted that all of this code and documentation is freely available online [38]. The Trusted Firmware Project is a 'not for profit' open source project hosted by Linaro Limited ("Linaro").

Arm Trusted Firmware uses the scaffolding provided by the A-Class devices 
to implement the key aspects of TrustZone, namely Trusted Boot and the Secure Monitor. There are currently over 30 platforms supported by Trusted Firmware and because the code is open source (BSD 3-clause), porting new platforms can be done by following many of the existing open source examples. Before we explore Arm Trusted Firmware, we must first understand how an Arm platform can be initialized in a secure state, specifically using Trusted Board Boot (TBB). TBB is based on two standards, the Arm Trusted Base System Architecture (TBSA) [5] and the Arm Trusted Board Boot Requirements (TBBR) [6]. Both of these specifications are client-based solutions and it is likely that server based solutions are in development internally at Arm.

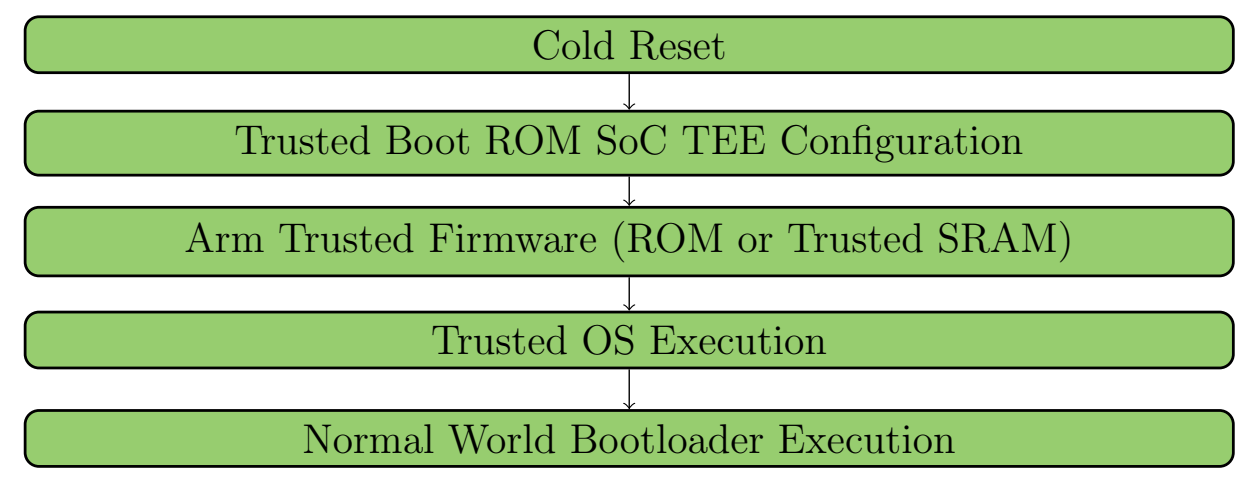

Figure 4.2: A significantly simplified boot flow for setting up a secure chain of trust on an Arm system running Trusted Firmware. Here we see the boot flow from a cold reset all the way to the Normal World bootloader which may load any untrusted OS kernel.

As soon as the SoC comes out of power-on-reset, execution happens in integrity protected memory like on-chip Boot ROM or Trusted SRAM. At this stage we have access to a Root of Trust Public Key Hash (ROTPK) located in one of the SoC's One Time Programmable (OTP) Efuse registers. These keys are usually SHA-256 and the Efuse is burned by the manufacturer to ensure the integrity of the keys. This secure ROM firmware code is often called the Bootloader Stage 1 or BL1, 
and it is responsible for checking the validity of the ROTPK. Using this key, BL1 can verify the hash of the next bootloader stage (BL2). The code in BL1 is the only code that must run in EL3, minimizing the amount of initialization code that must run at this critical privilege level.

Once in BL2, the code is executing in Secure World EL2 and the firmware can use the ROTPK to extract the Trusted World ROTPK and the Normal World ROTPK, which are used in turn to validate the Secure World Trusted OS hash as well as the Normal World Untrusted OS hash. All of these keys and hashes are included as extensions to the x.509 standard format, however there is no need for a valid Certified Authority (CA) certificate, as we are verifying the contents of the certificates and not the validity of a certificate issuer. BL2 also does some RAM initialization before it passes off to BL3 where the Secure Monitor is implemented.

This Secure Monitor runs in EL3 and is responsible for loading both the Secure OS as well as the Normal World bootloader. This bootloader might be UBoot or some Unified Extensible Firmware Interface (UEFI) implementation like TianoCore. This Secure Monitor stays resident in memory during the life of the system and will manage the interactions between the Normal and Secure Worlds. All of these stages (BL1 - BL3) of TBB are implemented by Arm's Trusted Firmware-A (TF-A) reference implementation.

Once the Trusted Firmware has initialized the system in a secure state, we have initialized two worlds, the Trusted World and the Normal World. It is perhaps easiest to think about the interaction between these two worlds in much the same way we think about making calls from user mode into kernel mode in Linux systems. In Linux systems, we take advantage of system calls (syscall) to bridge a trust boundary between the kernel's concerns like interacting with a network card 


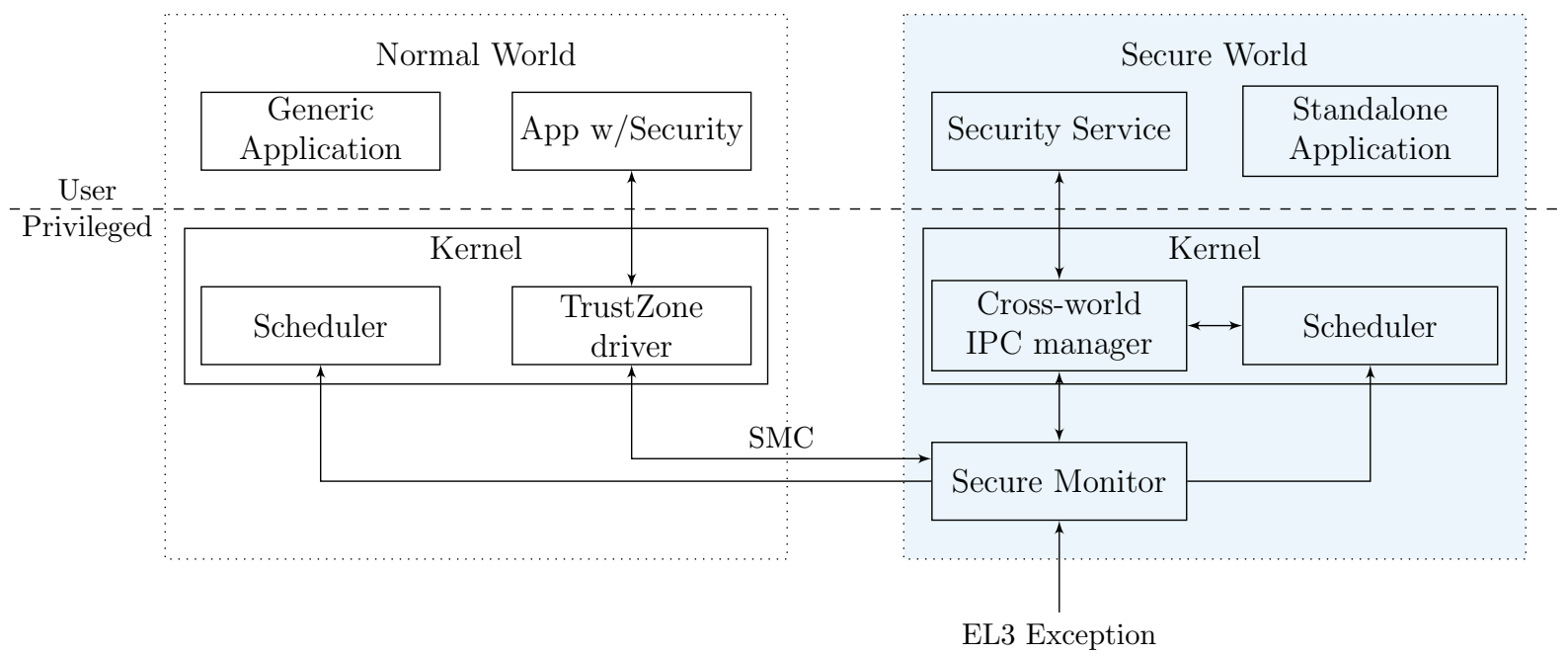

Figure 4.3: Secure World implementation using ARM TrustZone. The SoC boots into the Secure World and a monitor is registered which acts as the interface between the "secure" and "normal" worlds. All EL3 exceptions are caught by the secure monitor, and there is a special EL3 exception called a "secure monitor call" which is used to switch the processor between the two worlds. Figure recreated and modified from the original [59].

and the user application's concerns like displaying a web page. At no point in this interaction should the user application code have access to the network card's buffers, however the kernel is able to read and write these buffers and mediate the flow of data to/from the client application. This "guarded" flow of data is similar to how memory in the Secure World is kept separate from the Normal World using an instruction which generates an exception called a Secure Monitor Call (SMC).

\subsection{TrustZone Attestation}

Local attestation using TrustZone is dependent on several measurements taken during the boot process. If the boot process is not secured then "all bets are off", and we cannot assure the integrity or confidentiality of the code or data inside the TEE. We will follow this boot flow step by step, and each step number is illustrated 
in Figure 4.4.

At power on, implicitly trusted code living in secure ROM or SRAM is executed. Since Boot Loader 1 (BL1) is responsible for authenticating the BL2 stage it verifies the Root of Trust (RoT) public key in the BL2 content certificate against the RoT public key stored in the hash (Figure 4.4 - Step 1). BL1 then verifies the BL2 content certificate using the enclosed RoT public key (Step 2). BL1 loads BL2 into memory and verifies the hash (Step 3) and execution is transferred to BL2 (Step 4). Since BL2 is responsible for authenticating all of the possible BL $3_{\mathrm{x}}$ stages (e.g. $\left.\mathrm{BL}_{1}, \mathrm{BL} 3_{2}, \mathrm{BL} 3_{3}\right)$, BL2 verifies the RoT public key in the certificate against RoT public key stored in the hash (Step 5). BL2 then verifies the certificate using its RoT public key and saves the trusted world (TW) and normal world (NW) public keys. BL2 uses the TW public key to verify the BL3 ${ }_{\mathrm{x}}$ certificate (Step 6) and verifies the BL3 $3_{\mathrm{x}}$ content certificate using the BL3 $3_{\mathrm{x}}$ public key (Step 7). BL2 can now extract and save the BL3 $3_{\mathrm{x}}$ hash used for $\mathrm{BL} 3_{\mathrm{x}}$ image verification (Step 8). BL2 verifies the BL3 3 key certificate using the NW public key (Step 9) and verifies the BL3 $3_{3}$ content certificate using the BL3 3 pub key(Step 10). BL2 extracts and saves the BL3 $3_{3}$ hash used for BL3 3 image verification (Step 11). Finally, execution is transferred to verified BL3 $3_{\mathrm{x}}$ (Step 12) and BL3 $3_{3}$ images (Step 13).

We have now securely booted, and our secure monitor provided by TF-A should be loaded into BL3 ${ }_{1}$ running in the highest privilege level of EL3. The trusted OS, Open-source Portable TEE (OP-TEE) is loaded into BL3 $3_{2}$ and runs at EL1. Any trusted applications will run in EL0 of the "secure world" built on top of this secure OS. A "normal world" bootloader like U-Boot or some UEFI implementation is loaded into $\mathrm{BL}_{3}$ and runs in the privilege level of EL2 ${ }^{3}$. An operating system

\footnotetext{
${ }^{3}$ Systems boot UEFI at EL2 allowing for a hypervisor aware OS to be loaded. Booting UEFI at EL1 is likely done within a hypervisor hosted guest OS, allowing a UEFI-compliant OS to be
} 


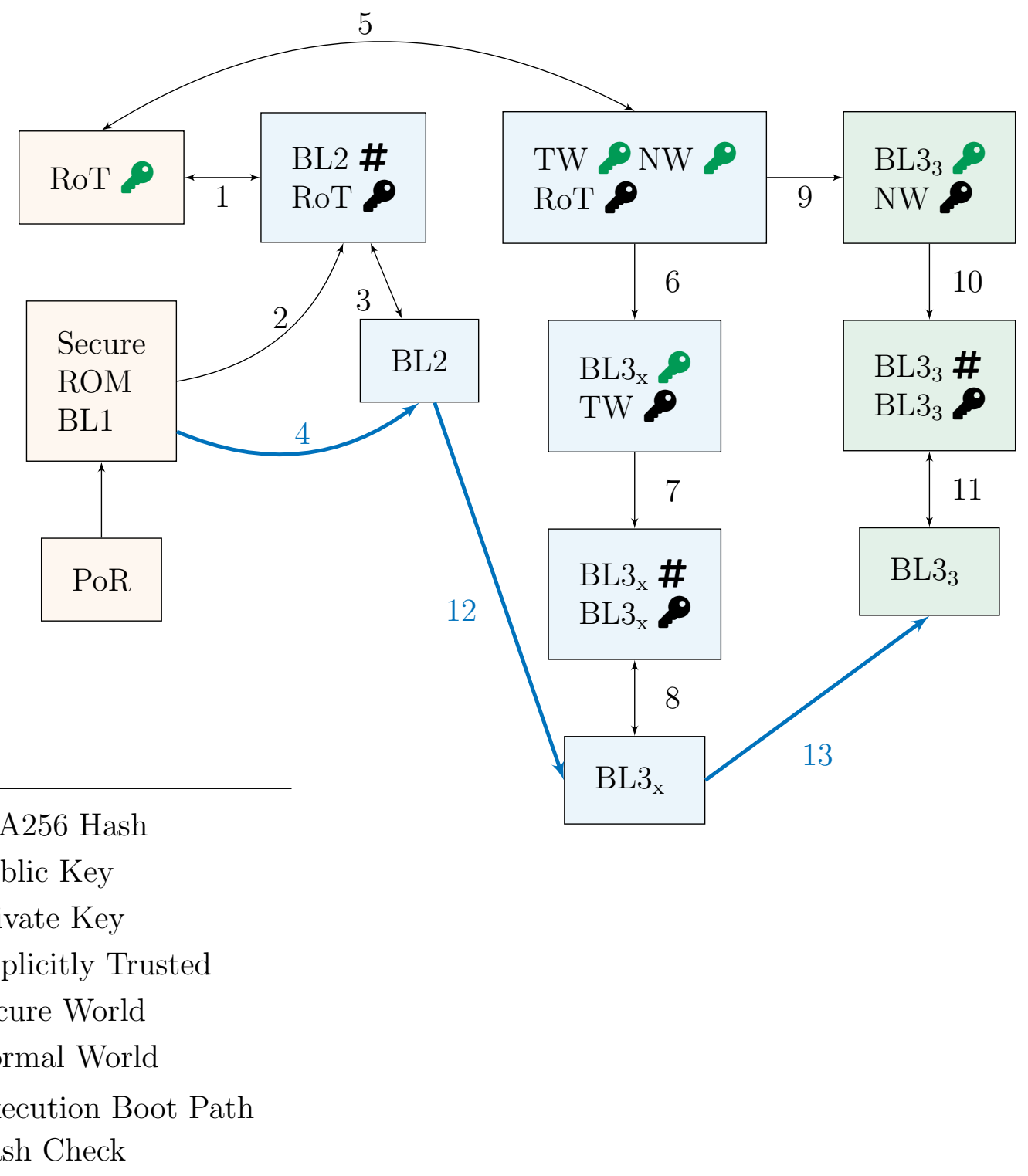

Figure 4.4: Trusted Board Boot (TBB) flow in Trusted Firmware-A (TFA) The boot flow begins with implicitly trusted keys and code living in some secure ROM or SRAM (BL1). Each stage of the boot is then verified against the RoT public key and each bootloader stage is hash checked. All this information can be found in the Arm TBBR-CLIENT documentation [6]. This diagram and flow description were recreated from a presentation by Matteo Carlini entitled "Secure Boot on ARM Systems: Building a Complete Chain of Trust Upon Existing Industry Standards Using Open-source Firmware". 
like Linux or Windows can now load their kernel into memory and will operate at privilege level EL1. Finally "normal world" applications can be loaded by the OS and run in privilege mode EL0. These applications can make SMCs with the OPTEE kernel driver in the non-secure world to act as a bridge to trusted applications running in the secure world.

OP-TEE will load Trusted Applications (TAs) into memory when a Rich Execution Environment (REE) application makes a request SMC with the corresponding UUID of the TA. For Linux-based systems, the TAs consist of an ELF binary, signed and possibly encrypted, named from the UUID of the TA. It is the responsibly of the trusted OS to load the TAs from the REE file system and to check the integrity of the TAs as part of the chain of trust. OP-TEE maintains a version database of all the TAs it has loaded and checks the version of each TA before loading. The version database prevents downgrading of the TA to a earlier, possibly insecure version.

Since loading a TA from the REE file system creates an inherently larger attack surface, there are two ways for OP-TEE to load applications from a more secure location. The first method is known as "early TA" and allows applications to be linked into the data section of the TEE core blob itself. The "early TA" method has two benefits: applications can be loaded from a known secure source and applications can be loaded before the normal world or its file system have been initialized. The other, more robust option is to load the application from secured storage, an OP-TEE implementation of the GlobalPlatform specification for Trusted Storage [25].

OP-TEE secure storage follows the GlobalPlatform TEE Core API document booted. 


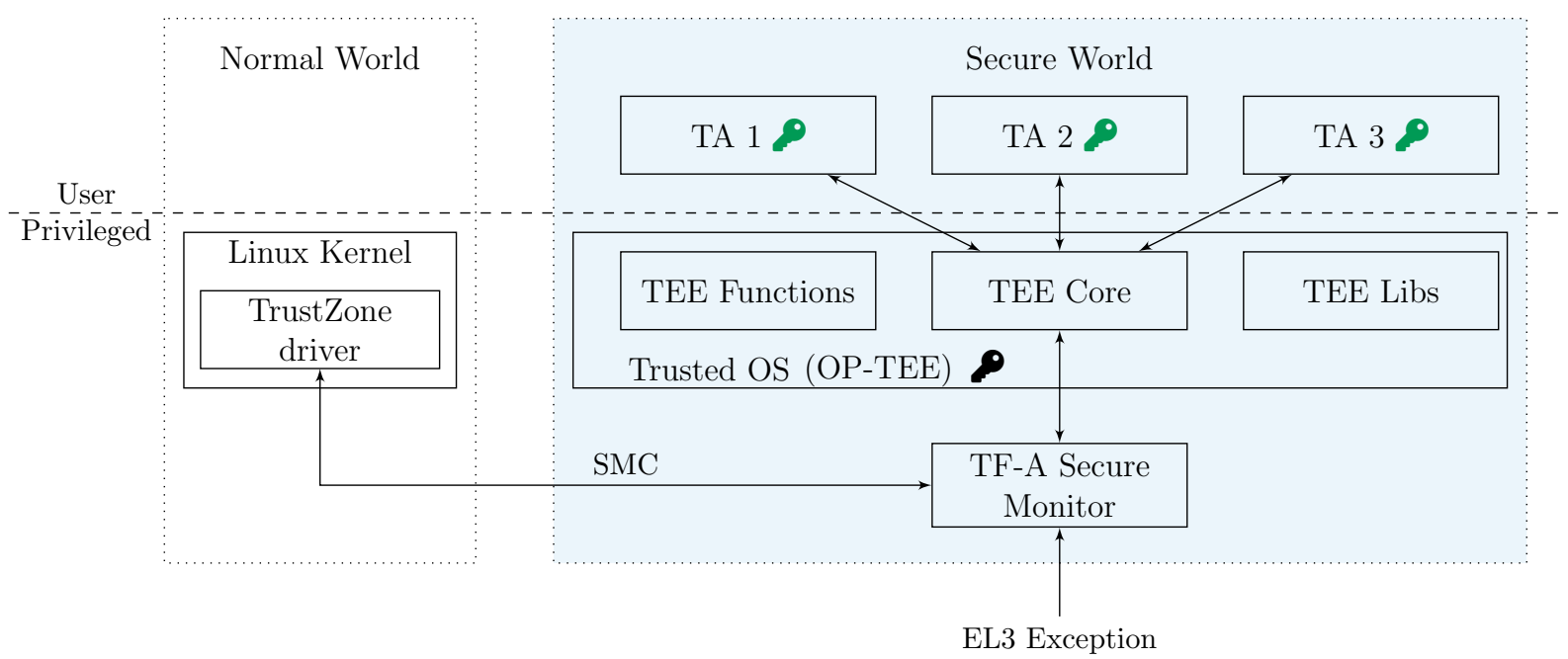

Figure 4.5: The OP-TEE Chain of Trust builds off the secure boot chain of trust. From Figure 4.4, we loaded the secure monitor into BL $3_{1}$ and our secure OS, OP-TEE, into BL3 $3_{2}$. With that chain of trust complete, we can now use the keys present in OP-TEE to load trusted applications into our TEE. The TEE functions and libraries actually live in the same area and are separated here only for convenience.

[26] defining "Trusted Storage API for Data and Keys" in section 5. The details of OP-TEE secure storage are beyond the scope of this thesis. The reader should understand that regardless of where the applications are loaded from, they are signed with a key from the trusted OS. OP-TEE Trusted Applications (TAs) are signed with the pre-generated 2048-bit RSA private key provided by the trusted OS developer as shown in Figure 4.5. These keys should be stored in a hardware security module like a Trusted Platform Module (TPM), however the security model for any given TA is left up to the trusted OS developer. Currently, OPTEE only supports one private key per trusted OS. As such, each TA will be signed with the same key. This step completes the chain of trust and we are able to assure that each step in loading the application, from PoR to loading the application into memory, has occurred in a way which preserves the integrity and confidentiality of 
the data as well as the integrity of the code.

The complexity of remote attestation on mobile platforms, specifically cell phones, has been well studied and understood for over a decade [43]. There have been many recent efforts towards a more formally proven type of remote attestation $[12,20]$ which can be used in IoT and embedded applications. However, Arm TrustZone as a framework leaves the implementation of remote attestation as an exercise for the platform vendor or manufacturer. We will return to this point in Chapter 6 when we present a comparison of our discussed TEEs.

In this chapter, we have shown how Arm has designed a framework on which hardware manufacturers and system vendors can hang an implementation of a TEE. Unlike with Intel SGX, and perhaps core to Arm's business model, many of the implementation details are left to the manufacturer. This allows for great flexibility at the cost of engineering resources and time. Next, we will explore RISC-V Physical Memory Protection (PMP), and describe a system which is open source from the ISA through to user space applications. As we will show, open source specifications allow for even greater flexibility than Arm TrustZone, though again at a greater cost. 


\section{Chapter 5}

\section{RISC-V Physical Memory Protection}

\subsection{The RISC-V Open Source ISA}

RISC-V is the fifth generation of reduced instruction set computer (RISC) processors, which have been in development since the 1980s at the University of California at Berkeley. Hence the name RISC-V has the "five" spelled out as a Roman numeral. One cannot talk about RISC-V's history without mentioning that of the MIPS ISA, which began in the same timeframe at Stanford in the 1980s. While MIPS was very popular in its own right, RISC was the inspiration for many ISAs including Sun Microsystems' SPARC line, DEC's Alpha line, Intel's i860 and i960 processors, and indeed the ARM processors that most of us now carry in our pockets.

RISC-V is by no means the first open source ISA. Sun Microsystems introduced the OpenSPARC project in 2005 using the GNU Public License (GPL) [2]. As many students of SoC architecture classes will know, the MIPS architecture was provided under an "open use" license in a pilot program by Wave Computing allowing its use in educational settings without licensing fees. ARM has partly opened its architecture allowing specific partners to proposed changes to the ISA they license. Many of these changes in industry practices reflect a growing need for openness in hardware. As Asanović notes, "While instruction set architectures (ISAs) may be proprietary for historical or business reasons, there is no good tech-

nical reason for the lack of free, open ISAs" [8]. RISC-V is also not an example of 
open source hardware as there is nothing inherent in the specifications encouraging the end product to be non-proprietary. However, it is certainly an enabler for open source hardware development by facilitating the sharing of ideas in the form of ISA extensions and hardware tooling.

Care must be taken not to assume that the value found in open source software can be simply replicated in the hardware world. However, there is value to be gained for certain. Many of the same foundations underpinning open source software development as well as many of the lessons learned by those communities can be applied in hardware. Red Hat's Patent Promise and the growing problem that international regulations introduce are just two examples [49]. The fundamental concept of extensibility is perhaps the most obvious cross-cutting concern between open source hardware and software. Tim O'Reilly mentions the importance of extensibility to the proliferation of open source software 1990's. As O'Reilly notes, "According to Linus Torvalds, Linux has succeeded at least in part because it followed good design principles, which allowed it to be extended in ways that he didn't envision when he started work on the kernel. Similarly, Larry Wall explains how he created Perl in such a way that its feature-set could evolve naturally, as human languages evolve, in response to the needs of its users." [46]. Key to RISC-V's success will be the proliferation of open source extensions, tools, and designs that anyone can use as a platform on which to produce and launch innovative hardware.

RISC-V Physical Memory Protection (PMP) is developed as part of the "TEE Task Group" of RISC-V International. While membership in RISC-V International is required to participate in the development of specifications, membership is free for individuals and open to anyone. Specifications are developed using public mailing lists, and a review of each specification is open to a public review period 
before ratification. The specifications themselves are stored on a public GitHub repository where anyone can view the text during its development. RISC-V PMP was added to the Privileged ISA Specification [63] in 2019. Several libraries have been introduced to take advantage of this technology including: Multizone [50], Sanctum [16], TIMBER-V [65], Mi6 [10], and Keystone Enclave [14, 36, 37]. This thesis will concentrate on Keystone Enclave as it is open source and still in active development.

\subsection{The RISC-V Memory Model}

An understanding of how RISC-V handles memory is required in order to understand how RISC-V PMP protects said memory. There are two current memory models available as part of the RISC-V specification. The first memory model presented in the specification is the RISC-V Weak Memory Order (RVWMO) model, "which is designed to provide flexibility for architects to build high-performance scalable designs while simultaneously supporting a tractable programming model" [62]. There is also the "Ztso" extension which gives us the RISC-V Total Store Ordering (RVTSO) memory consistency model. One can think of RVTSO as the "less flexible" model when compared to RVWMO. We will only cover RVWMO briefly here as knowledge of RVTSO is not required to understand PMP, nor is a deep understanding of the RVWMO required.

The memory model of any instruction set architecture defines the values returned by a load. As such, we must first understand what we are loading into memory. RISC-V hardware threads, which are referred to in the specification as "harts" [62], have a byte-addressable address space of $2^{\text {XLEN }}$ bytes for all memory accesses. Here, XLEN refers to the width of a register in either 32 or 64 bits. 
Words are defined as having 32 bits. Halfwords are 16 bits, doublewords are 64 bits ( 8 bytes), and so on. The address space can be thought of as a ring, so that the last memory address is adjacent to the first. Memory instructions will simply wrap around the space ignoring that they have effectively "walked off the end". The specification leaves room for virtual memory by allowing for both explicit and implicit stores and loads. Chapter 3 of the RISC-V Unprivileged Specification [62] introduces the "Zifencei" extension, which defines a fence instruction that explicitly synchronizes writes to instruction memory and instruction fetches on the same hart. The order in which these loads and stores happen is up to the implementation, and the rules for consistency are defined in the memory model.

The microarchitecture implementing any given memory model is only required by the architecture to follow the rules set forth in the model. This model does not set any other regulation on how the implementation achieves those rules, be the implementation speculative or not, in order or out of order, multithreaded or not, etc. As such, the RVWMO starts by defining a set of primitives it uses to define the model. The base primitives are load and store, defined in Chapter 8 which covers the "A" Standard Extension for Atomic Instructions [62]. It then uses a combination of axiomatic and operational semantics to define how memory consistency should be achieved.

The RVWMO model defines the syntactic dependencies of memory operations. This is essentially a way to understand what the differences are between memory operations and the instructions which generate those operations. The memory model then defines thirteen rules that allow for the program order of each hart to be consistent with the global order of all operations, called the "preserved program order". The rules cover overlapping address ordering, explicit synchronization, 
syntactic dependencies, and pipeline dependencies. The model then defines three axioms: the Load Value Axiom, the Atomicity Axiom, and the Progress Axiom. Any implementation which follows the RVWMO memory model must conform to the thirteen rules and satisfy the three axioms.

\subsection{RISC-V Physical Memory Attributes}

RISC-V system's physical memory map has various properties and capabilities that are described in detail in the Privileged Specification [63] and referred to as Physical Memory Attributes (PMA). These attributes determine things like read, write, and execute permissions of a specific region of physical memory. Most systems built on the RISC-V architecture will require that the PMAs are checked later in the pipeline and that this check is done in hardware. This is in contrast to other architectures where these types of checks are done in virtual page tables where the Translation Lookaside Buffer (TLB) contains the information the pipeline needs regarding these attributes. RISC-V systems call the mechanism for making these checks the "PMA checker" and many physical attributes will be hardwired into the checker when designing the chip. For those attributes that are not known at design time there are special platform specific control registers that can be configured at runtime.

Memory regions are given attributes based on if they are part of main memory or part of I/O. The access width of a region can be anything from 8-bit byte to long multi-word bursts. As the specification states, "Complex atomic memory operations on a single memory word or doubleword are performed with the load-reserved (LR) and store-conditional (SC) instructions." [63]. RISC-V also allows for special atomic memory operations (AMOs) which are instructions that 
perform operations for multiprocessor synchronization. A given PMA will contain information regarding which atomic operations are allowed for a specific region of memory. Table 5.1 lists the AMO available to I/O as of this writing.

\begin{tabular}{|l|l|}
\hline AMO Class & Supported Operations \\
\hline AMONone & None \\
AMOSwap & amoswap \\
AMOLogical & above + amoand, amoor, amoxor \\
AMOArithmetic & above + amoadd, amomin, amomax, amominu, amomaxu \\
\hline
\end{tabular}

Table 5.1: Classes of AMOs supported by I/O regions. Reproduced from the original RISC-V Privileged Specification [63].

As mentioned earlier, regions of memory will be classified either as main memory or as I/O, and this must be considered by the FENCE instruction when ordering memory. Regardless of whether the memory model used is RVWMO or RVTSO, memory regions may be classified as either having relaxed or strong ordering. Strongly ordered memory regions use a "channel" mechanism to guarantee ordering. Using PMAs, systems can decide to set the type of ordering dynamically or not. The specification requires that all regions of memory be coherent, such that any change made by one agent to a memory region must eventually be visible other agents of the system. Cacheability is left up to the platforms, however three types are called out: "master-private, shared, and slave-private" [63]. PMAs will describe the specific cache features of each region as well as if the region is idempotent. 


\begin{tabular}{|l|l|}
\hline Processor Mode & Description \\
\hline U Mode & User Processor Mode \\
\hline S Mode & Supervisor Processor Mode \\
\hline H Mode & Hypervisor Processor Mode (Draft) \\
\hline M Mode & Machine Processor Mode \\
\hline
\end{tabular}

Table 5.2: RISC-V Privilege Level Mapping is similar to Arm's exception level mapping in that we have four modes. The most privileged machine mode is the only required mode.

\subsection{RISC-V Physical Memory Protection}

Unlike PMAs, RISC-V Physical Memory Protection (PMP) consists of a set of configurations that can be changed dynamically during runtime. The privileged specification describes a "PMP unit" as a set of "per-hart machine-mode control registers to allow physical memory access privileges (read, write, execute) to be specified for each physical memory region." [63]. These registers must be checked in parallel with the PMA attributes described in the previous section.

As we discussed in the previous two chapters, processor privilege levels or "modes" are a critical foundation to our security model. RISC-V PMP allows for specific registers that are only available to the highest privileged machine-mode or m-mode. The different processor modes for RISC-V are described briefly in Table 5.2. Note that $\mathrm{H}$ Mode is still only in the draft state, and is not applicable when discussing PMP. While PMP is available in both 64 bit and 32 bit versions, we will only describe 32 bit configurations in this thesis. It is enough for our comparison that we understand a 64 bit implementation to be possible and to hold to the same or standards as the 32 bit version. 
(a)

\begin{tabular}{|c|c|c|c|c|c|}
\hline 31 & 23 & 15 & 8 & 0 & \\
\hline pmp3cfg & pmp2cfg & pmp1cfg & & pmp0cfg & pmpcfg0 \\
\hline 8 & 8 & 8 & & 8 & \\
\hline 31 & 23 & 15 & 8 & 0 & \\
\hline pmp7cfg & pmp6cfg & pmp5cfg & & pmp4cfg & pmpcfg1 \\
\hline
\end{tabular}

\begin{tabular}{|c|c|c|c|c|c|}
\hline 31 & 23 & 161 & 15 & 0 & \\
\hline pmp11cfg & pmp10cfg & & pmp9cfg & pmp8cfg & pmpcfg2 \\
\hline 8 & 8 & & 8 & 8 & \\
\hline 31 & 23 & 161 & 15 & 0 & \\
\hline pmp15cfg & pmp14cfg & & pmp13cfg & pmp12cfg & pmpcfg3 \\
\hline
\end{tabular}

\begin{tabular}{|c|c|}
\hline 31 & 0 \\
\hline & address[33:2] (WARL) \\
\hline 32
\end{tabular}

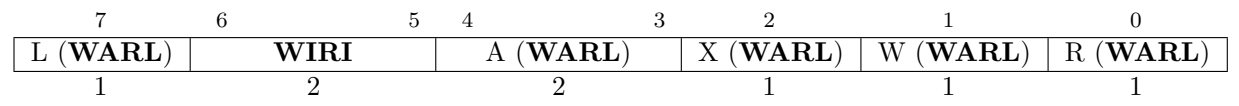

Figure 5.1: RV32 PMP CSR layout and format as well as the address register format. Figure 5.1a describes the RV32 PMP configuration CSR layout. Figure 5.1b is the PMP address register format for RV32. Lastly, Figure 5.1c shows the PMP configuration register format. Figures reproduced from the RISC$\mathrm{V}$ privileged specification [63].

There are currently up to 16 PMP configuration registers available in both 32 and 64 bit modes and their layout for 32 bit is shown in Figure 5.1a. Next, in Figure 5.1c, we see a description of the layout for those configuration registers where WARL is Write-Any Read-Legal and WIRI is Write-Ignored ReadIgnored. This is to say that the two bits 5 and 6 are simply ignored in this register as they are reserved. All the other bits will follow the RISC-V common WARL field rules as defined in the CSR section of the specification. There is also an address register format defined in Figure 5.1b, which is the starting address of the PMP region and encodes bits 33-2 of the 34-bit physical address length" ${ }^{1}$ No "stop address" is required, and if only one PMP region is defined, it

\footnotetext{
${ }^{1}$ Per the RISC-V specification, "The Sv32 page-based virtual-memory scheme described in Section 4.3 supports 34-bit physical addresses for RV32, so the PMP scheme must support addresses wider than XLEN for RV32." [63].
} 
will encompass the entire memory space. Indeed when many systems boot they may choose to create a single PMP region with read/write/execute enabled for all modes as a default setting.

The "L" bit of the configuration register defines if that region of memory is locked and cannot be read, written, or executed from, regardless of the processor mode. A reset is required for m-mode to once again control that CSR and allow that region's permissions to change. As mentioned, bits 6 and 5 are reserved. Bits 4 and 3 define the type of address matching scheme that will be used to match the store or load address range against the PMP address register. The options for this two bit value are OFF, Top of Range (TOR), Naturally Aligned 4-byte region (NA4), or Naturally Aligned Power of Two (NAPOT). If set to NAPOT the granularity can be set by the system to any NAPOT value greater than or equal to 8. The last three bits are set to 1 or 0 for read, write, and execute, with 1 meaning "enabled" and 0 meaning "disabled". These bits currently apply to both S Mode and $\mathrm{U}$ Mode, though we will discuss the future of S Mode PMP in a following section.

Using these configuration registers and address registers, RISC-V systems are able to create regions of memory with simple read, write, and execute privileges enforced by hardware. This allows systems to build secured areas of memory similar to that of both Intel Software Guard Extensions (SGX) and Arm TrustZone. However, without a framework on which to build these secured areas of memory, the specification only enables hardware engineers to create a foundation on which to build a Trusted Execution Environment (TEE). We will now examine Keystone Enclave, an open source framework for building hardware TEEs using RISC-V PMP as the foundation. 


\subsection{Keystone Enclave}

One of the first TEE solutions for RISC-V PMP came out of the MIT Computer Science \& Artificial Intelligence Laboratory (CSAIL) and a project called Sanctum [16]. This work was done just before the RISC-V PMP task group ratified the first version of their specification for PMP in 2017. As such it was more of a prototype and required non-standard extensions to the RISC-V core. Keystone Enclave came out of efforts of researchers at UC Berkeley and takes advantage of the ratified PMP specification.

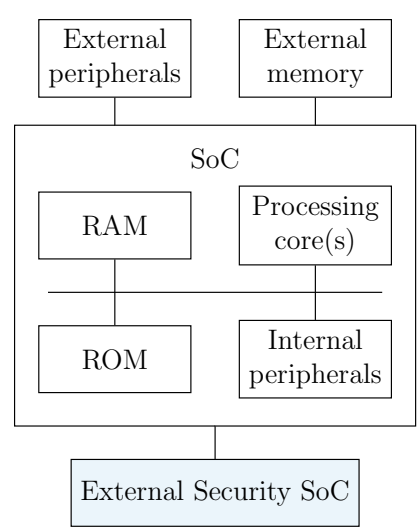

a) External secure element

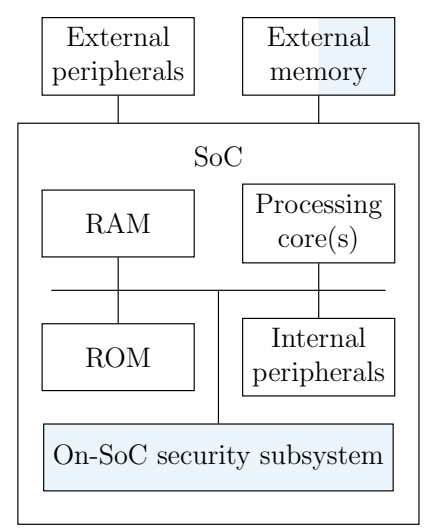

b) Embedded secure element

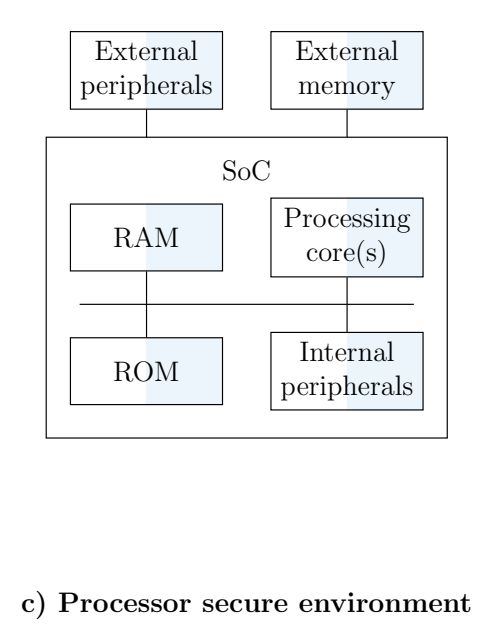

c) Processor secure environment

Figure 5.2: A high level overview of possible RISC-V PMP configurations. RISC-V is highly customize and we can imagine many different possible solutions when building a platform. Shaded areas imply that some logic is present which allows PMP to secure the TEE. Reproduced as a simplified version of Figure 2-3 of the GlobalPlatform specification [25] as well as Teschke's rendering [59].

As detailed in Figure 5.3, Keystone is a full software stack containing a firmware base which launches both trusted and untrusted "runtimes". These "runtimes" then launch both trusted, isolated applications into the enclave as well as untrusted 
applications into the user space of a Linux-based operating system. Keystone defines a set of TEE primitives [36]: Secure Boot, a "Secure Source of Randomness", and Remote Attestation. Keystone builds its framework off these primitives and allows for customization of their firmware which they call a secure monitor (SM). The SM is used to "enforce TEE guarantees on the platform" [37], and does so by taking advantage of key properties of RISC-V machine mode. These properties include machine mode's programmability, its ability to delegate interrupts, and its ability to manage memory access using PMP.

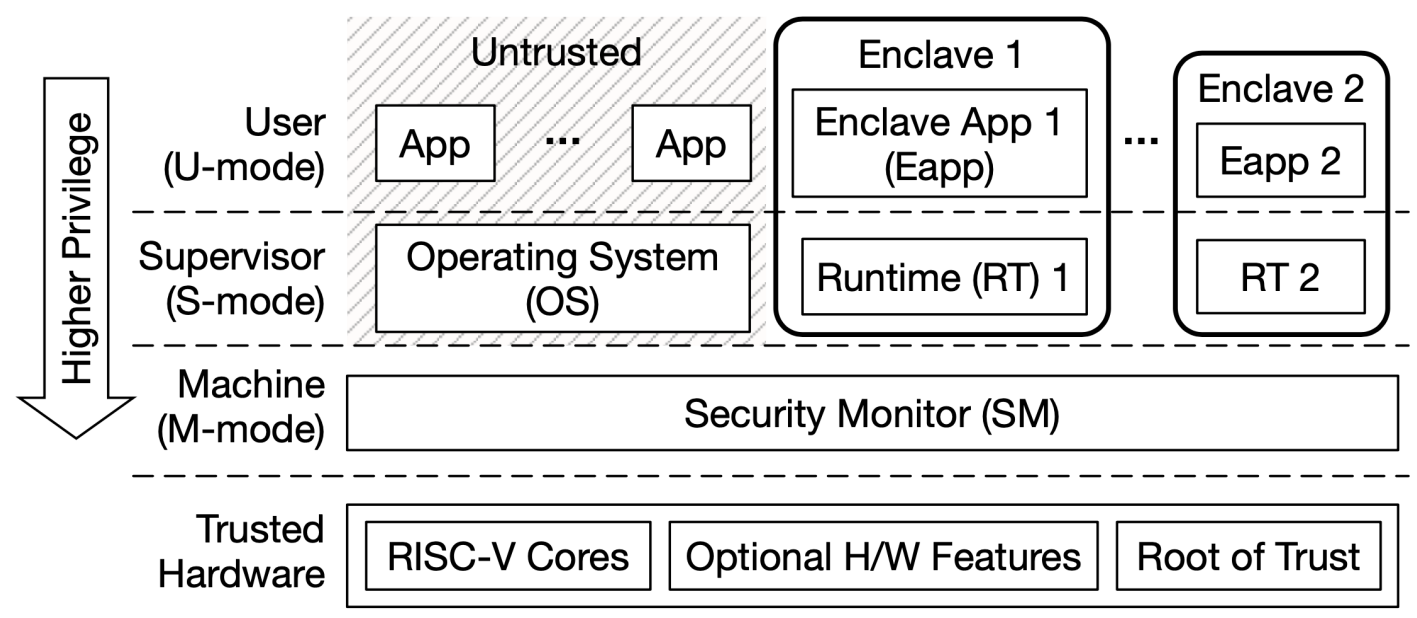

Figure 5.3: A high level overview of a Keystone Enclave system. The Keystone codebase includes the security monitor, enclave runtime, enclave application, Linux as an untrusted OS, and untrusted host applications. Reprinted from Figure $1[37]$.

There are two key features of RISC-V PMP which Keystone takes advantage of in building their framework. The first is memory isolation which allows the secure monitor (SM) running in machine mode to restrict access to the enclave's memory. The second feature is the ability for PMP to allow Keystone Enclave secure applications to use multiple hardware threads in executing code. As Lee notes, "RISC-V 
provides per-hardware-thread views of physical memory via machine-mode and PMP registers. Using RISC-V thus allows multiple concurrent and potentially multi-threaded enclaves to access disjoint memory partitions while also opening up supervisor-mode and the MMU for enclave use." [36].

PMP registers

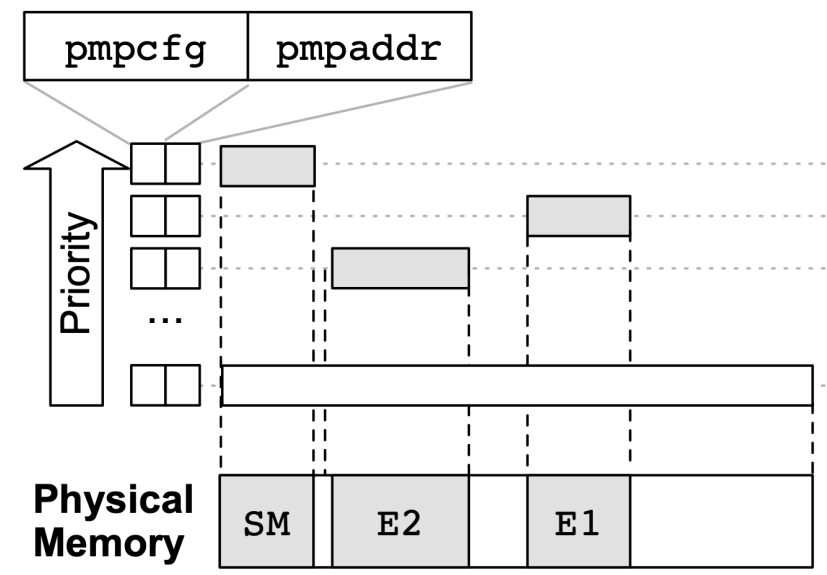

Untrusted Context pmpcfg perm.

$\square \mathrm{rwx}=000$

$\square \mathrm{rwx}=111$

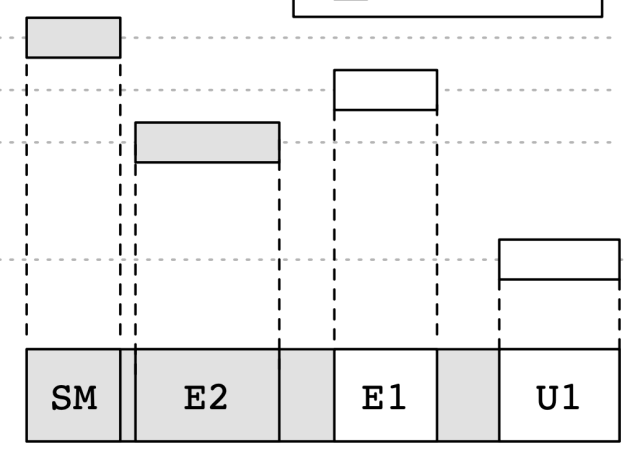

Enclave (E1) Context

Figure 5.4: Keystone memory isolation using RISC-V PMP as a primitive. Keystone separates each untrusted and trusted application into its own memory space. The secure monitor (SM) is responsible for ensuring that the proper pmpcfg and pmpaddr configurations are applied with the correct permissions. Reprinted from Figure 2 [37].

Looking at Figure 5.4, we can see the way in which Keystone Enclave restricts access to several sections of memory. Keep in mind that the priority of these regions are read from top to bottom with the top being the highest priority and the bottom the lowest. In the untrusted context a lowest priority area is created in order to give read, write, and execute permission to both supervisor and user modes. This allows the untrusted OS, in this case Linux, to run applications anywhere outside the secure monitor and enclave regions. The security monitor sets its own region configuration as the highest priority such that no other code 
should be allowed access to the secure monitor's region. When a context switch is made into machine mode (E1 Context), the processor begins executing enclave code. The secure monitor switches the enclave (E1) and user application (U1) configuration bits. Those regions of memory now have read, write, and execute privileges until the context switches back to the "Untrusted Context".

The memory management of these enclaves is controlled by the Keystone Enclave runtime called "Eyrie" which can be seen in the high level diagram in Figure 5.3 as RT1 and RT2. Eyrie runs in Supervisor Mode and not only performs page table operations, but also allows for dynamically resizing the enclave. The security monitor has a plugin which allows for pages which must be evicted from secure memory to be encrypted such that the confidentiality of the data can be assured as it leaves the trusted region. This plugable interface of the secure monitor and Eyrie runtime, along with the open source nature of the project as a whole, allows for great flexibility. For example, the secure monitor has an "on-chip memory plugin" [37] that allows for use of a scratchpad memory onto which the enclave can be loaded. The Eyrie runtime has a plugin which allows an enclave to communicate with host applications in a secure manner using a shared buffer. While syscalls are not allowed inside the enclave itself, the secure monitor can either proxy those syscalls to the untrusted application, or run them in a trusted context if appropriate (e.g. mmap, brk, getrandom) [37].

While Keystone Enclave provides many features that we require in our definition of a TEE, some features are notably left to the user. Remote attestation is possible, however as the Keystone authors note, "Key distribution, revocation, attestation services, and anonymous attestation are orthogonal challenges" [37]. There are projects currently working on the problem of remote attestation on 
RISC-V hardware [56], however these efforts are still in their infancy. Keystone Enclaves provide a method of local attestation which makes use of provisioned keys. Enclaves can, during runtime, request signed attestation from the secure monitor in a kind of attestation report similar to that found in Sanctum [35]. This local attestation is built off of a Secure Boot process that is very similar to TrustZone's model, and includes the use of hardware key management and cryptographic engines. Efforts to continue work on secure boot RISC-V systems is the focus of research [29], but no dominant technology currently exists as a de facto standard.

\subsection{Future Extensions of RISC-V Memory Protection}

Three additional features of RISC-V PMP will doubtless play a role in future work done on Keystone Enclave or any RISC-V based TEE framework. The first is a draft of an Extended Physical Memory Protection (ePMP) specification which details how PMP will be able to secure user mode code from being executed by machine mode. Attacking user mode from a more privileged mode is a well known security vulnerability first address in Intel Ivy Bridge processors about a decade ago [53]. Seagate recently announced their completion of a chip which takes advantage of this draft ePMP features. The draft ePMP specification is currently supported inside the Spike simulator, which is the de facto functional simulator for RISC$\mathrm{V}[7]$. ePMP has also been implemented in open source hardware cores like the Ibex core from lowRISC [39] as well as the open source Root of Trust (RoT) OpenTitan [28].

The second additional feature, S-Mode Physical Memory Protection (sPMP), is also in draft status as of this writing. sPMP will provide a way to ensure that systems without virtual memory can take full advantage of PMP. Currently the 
separation between S Mode and U Mode is done in virtual memory. This extension will allow for PMP configuration registers specifically reserved for S Mode, moving that protection from virtual memory into hardware registers. While sPMP is not implemented yet in any simulators or publicly available hardware, sPMP will likely see more development this year as the specification nears ratification.

The final additional feature, the draft specification for I/O Physical Memory Protection (IOPMP), aims to provide memory protection for additional memory masters such as Direct Memory Access (DMA). These memory masters will all share one IOPMP unit that will allow for both single core and multi-cores systems, support error reporting, and support a scalable number of IOPMP entries. The current draft specification defines an address table and IOPMP configuration registers which provide address matching for each of the IOPMP addresses.

All three of these extensions, ePMP, sPMP, and IOPMP are in various stages of ratification by RISC-V International. As such, their features are still in flux to different degrees. However, ePMP, sPMP, and IOPMP show the direction that RISC-V is heading in terms of TEE development as an attempt to provide as many if not more features than currently available TEE technologies.

We have now covered three different implementations of a Trusted Execution Environment (TEE). These high level descriptions will act as a general reference in the next chapter where we will provide a method for comparing these technologies. The method we present can be applied to any set of TEEs regardless of their complexity or their intended target hardware. The three examples of a TEE have provided a foundation for the systematic and rigorous comparison that will be detailed in the following chapter. 


\section{Chapter 6}

\section{Trusted Execution Environment Comparisons}

\subsection{A Method for Comparing TEEs}

When we compare Intel Software Guard Extensions (SGX), Arm TrustZone, RISCV Physical Memory Protection (PMP), or any group of Trusted Execution Environments (TEEs) we must first consider what we are comparing. As such we define any given TEE based on a set of properties that the TEE must implement or optionally might implement. We will make the assumption that the end goal of the comparison is to choose a TEE that best meets the needs of the system architect. As such, we must take the system architect's needs into consideration in choosing which properties that we will compare. An architect designing an embedded system with no access to networking will certainly not require traditional remote attestation, as an example.

As mentioned in Chapter 1, we will use the properties of a TEE as defined by the Confidential Computing Consortium (CCC) [58]. The CCC is an open source project that brings together hardware vendors, cloud providers, and software developers to accelerate the adoption of TEE technologies and standards. They define code integrity, data integrity, and data confidentiality as three required properties of any hardware TEE. All three of the TEE technologies which we have summarized in the preceding chapters provide these three required features in different ways. The CCC also discusses more advanced features like code confidentiality, authenticated launch, programmability, attestation, and recoverability, which can be considered optional properties of a TEE. As a final option of a TEE, we will 
include extensibility, which will be discussed in detail shortly.

Notably absent from our list of required properties is code confidentiality. There are several reasons why one might want code to be confidential. Code may contain proprietary algorithms for machine learning or other intellectual property. Likewise code may contain information about health or safety systems which must remain confidential to assure functional safety. On the other hand, the code that runs inside the TEE may be open source, making the feature of code confidentiality nonessential. As such, code confidentiality is considered an optional property by our definition.

By authenticated launch, we mean a mechanism by which the TEE can prevent a host application from loading, or limit its functionality based on a set of criteria or security model. One can think of this in terms of a banking application on a cell phone. If any stage of the attestation process fails, we want to recognize that code integrity can no longer be assured. We may want to fail to load the banking application with appropriate errors if code integrity is compromised. However, we may want some of the features of the banking application available, while restricting others. Authenticated launch may restrict withdrawing funds from an account while allowing the application to provide text messaging with technical support in order to resolve the problem.

By programmability, we mean the ability of a TEE to be programmed with code that is unrestricted and able to be changed by the end user. This is apposed to a TEE with a limited set of available functions or one that is pre-programmed by the manufacturer or system vendor. Programmability will be limited by the type of instructions which are allowed to be executed from inside the TEE. The size of the region of code that is allowed to be used by the TEE may also be restricted. 
Lastly, the way in which code running inside the TEE is allowed to communicate with code running outside the TEE is also a consideration of programmability. We will consider a TEE programmable to different degrees based on these limitations.

By attestation, we will use the definition by the CCC provided in Section 6 of their paper [58]. The attestation workflow starts with a secure connection between the two parties, identified as the verifier and the attester. The verifier must first challenge the attester to initiate the check. The challenge from the verifier signals to the attester that it must communicate with trusted hardware to request a measurement of the TEE. The trusted hardware is responsible for the measurement value as well as any certification of origin. The attester can then return these values to the verifier. The verifier will check any signatures provided based on the security model. Attestation may be performed locally on the host machine. If the verifier is on a remote machine to that of the attester we would consider that to be remote attestation. Attestation should take advantage of a Root of Trust (RoT), however that root of trust may be either hardware or software based. If a given system contains a hardware RoT we can consider that system more secure by the same definition we gave earlier of a hardware TEE.

By recoverability, we mean a way for the TEE to "roll back" to some known good state should a failure occur while preforming validation on the integrity or confidentiality of the system. The goal of recoverability is to re-establish trust in the Trusted Compute Base (TCB). Part of the TCB may not be easily recoverable. For example, if the platform firmware loaded onto an Intel SGX system is found to be untrustworthy, a simple firmware update may not be capable of removing malicious code. However, if the code running inside the SGX enclave is found to be untrustworthy, simply reloading that code from its original source may be enough 
to reliably restore trust in the application.

Finally, we add extensibility as the final optional property of a TEE. Extensibility was not defined in the CCC document, however extensibility can be observed to some extent in all three TEE technologies we have covered. We define extensibility as the ability to customize the deployment and management of the TEE environment, including hardware, firmware, and software. This property comes at the cost of supporting those customizations. As Linus's Law states, "Given enough eyeballs, all bugs are shallow" [52]. Regardless, the burden of support for customization must be a consideration, and a healthy open source project is no small feat.

While these properties are a good starting point for analysis and comparison, we must break these properties down further in order to engage in a more rigorous comparison. Authenticated launch can be broken up into two parts: the ability to prevent application launch, or the ability to launch applications with limited functionality. Programmability will be broken into three types: the size available to the TCB, the number of unique trusted applications one can run, and the number trusted OS's one can run to manage these applications. Attestation can be divided into three parts, with remote attestation, local attestation, and if a hardware root of trust is available. Recoverability will be divided into three parts describing system which allow: revocation of keys or other components, ability to recover the TCB using a hardware RoT, and finally if re-issuing keys is possible without the need for re-provisioning. Extensibility will be broken up into three parts: hardware, firmware, and software. We will consider the levels of extensibility as either "highly extensible", "somewhat extensible", or "not extensible". 


\subsection{Mapping Data Points}

This method uses tables to chart the comparison of these properties and subproperties we have discussed. In the following Tables 6.1, 6.2, and 6.3, we give example tables for the three technologies discussed in the previous chapters. The first Table 6.1 covers all six of the optional properties of a TEE that we have discussed in this chapter. Rather than grading each implementation of the property in a simple "good, better, best" fashion, we list if the property is possible in the given solution, and if so, the dependency of that property. Next, in Tables 6.2 and 6.3 , we pick two of the optional properties of a TEE and divide them into the smaller pieces we mentioned in the previous section. A more rigorous comparison can then be made on the details of each property.

This thesis only breaks down two of the optional properties of a TEE into greater detailed tables. It is left up to the reader to continue this process for the additional properties. Also, as a reminder to the reader, the data provided in this

thesis can and will change drastically over time. All of the following tables are meant as examples to the process of comparison and should not be considered as complete and wholly valid data sources.

Noting that the optional property of a TEE is available and listing required dependencies is helpful, however we also make a point to parenthetically note an example dependency where applicable. At the highest level, as in Table 6.1, this can be quite broad indeed. For example, attestation is possible with TrustZone and is provided locally by firmware or remotely by SoC manufacturers like Qualcomm. This is further illustrated in Table 6.2, where we show that local attestation can be achieved merely by using the appropriate firmware, however to achieve remote attestation, a system like Qualcomm's MSM8974 Snapdragon-based platform is 
required [57]. Again, note that we are using quite an old example with Qualcomm's MSM8974, circa 2016. Readers wishing to implement this method of comparison would be wise to chose current security platforms. Likewise, we list Intel as the only current solution to providing remote attestation for SGX solutions, however it is highly likely that other solutions are available.

The final Table 6.3 compares extensibility and requires we provide context. The table works from high level features like the Software Development Kit (SDK), to low level features like the actual HDL used to generate the TEE. As mentioned earlier, extensibility does not require that the system in question be open source. However, since open source options exist, and those systems are inherently extensible, we have listed those where possible. To say that the firmware is open source and therefore extensible does not provide enough explanation, and we should be more precise with our definition. Looking at TrustZone, the trusted OS provided by Open-source Portable TEE (OP-TEE) and secure boot provided by Trusted Firmware-A (TF-A) allow a high level of customization and extension. Likewise the Keystone project allows developers to extend its feature set with a set of "plugable interfaces" [37]. While Intel and others have worked to provide open source and extensible firmware for their platforms, the goal of those firmware projects is not to provide systems with a way to extend the features provided by Intel SGX. Therefore, while firmware available for SGX is indeed open source, it is not specifically designed with extensibility for SGX in mind.

As mentioned earlier, extensible systems as described in Table 6.3 may lack vendor-supplied support options. While Intel systems may seem like the more ridged of the three options, Intel aims to provide a high level of support for their 
large cloud and data center customers. Providing adequate support to those customers is a delicate balance between the ability to customize Intel systems and the need to provide uniformly high quality support to those customers. One can certainly build a RISC-V system which is completely customized from the HDL to the software SDK, but the responsibility of supporting that system will lie with the entity which customized it or the open source community built up around that system.

\begin{tabular}{|c|c|c|c|}
\hline & Intel SGX & Arm TrustZone & RISC-V PMP \\
\hline Attestation & $\begin{array}{l}\text { Platform } \\
\text { dependent (Intel, } \\
\text { Supermicro) }\end{array}$ & $\begin{array}{l}\text { SoC } \\
\text { manufacturer } \\
\text { dependent } \\
\text { (Qualcomm) }\end{array}$ & $\begin{array}{l}\text { Firmware } \\
\text { (Keystone, } \\
\text { Sanctum) }\end{array}$ \\
\hline $\begin{array}{l}\text { Authenticated } \\
\text { Launch }\end{array}$ & $\begin{array}{l}\text { Platform } \\
\text { dependent (Intel) }\end{array}$ & $\begin{array}{l}\text { Firmware } \\
\text { dependent } \\
(\mathrm{TF}-\mathrm{A})\end{array}$ & $\begin{array}{l}\text { Firmware } \\
\text { dependent (none, } \\
\text { currently) }\end{array}$ \\
\hline $\begin{array}{l}\text { Code } \\
\text { Confidentiality }\end{array}$ & Provided by SGX & $\begin{array}{l}\text { Platform } \\
\text { Dependent } \\
\text { (MCIMX8M- } \\
\text { EVKB) }\end{array}$ & Provided by PMP \\
\hline Programmable & Provided by SGX & $\begin{array}{l}\text { Provided by } \\
\text { TrustZone }\end{array}$ & $\begin{array}{l}\text { Firmware } \\
\text { (Keystone) }\end{array}$ \\
\hline Recoverable & $\begin{array}{l}\text { Platform } \\
\text { dependent } \\
\text { (Supermicro) }\end{array}$ & $\begin{array}{l}\text { Firmware } \\
\text { dependent } \\
(\mathrm{TF}-\mathrm{A})\end{array}$ & $\begin{array}{l}\text { Firmware } \\
\text { dependent (none, } \\
\text { currently) }\end{array}$ \\
\hline Extensible & Not extensible & $\begin{array}{l}\text { Somewhat } \\
\text { extensible }\end{array}$ & Highly extensible \\
\hline
\end{tabular}

Table 6.1: Several optional TEE features and the dependencies of those features. Optional features of a TEE can be provided by the technology itself, by the chip manufacturer, by the platform vendor, or by the firmware. In turn, the firmware can be provided to the end user by the hardware manufacturer, by the platform vendor, or can be custom. We mention some possible examples of platforms manufacturers or firmware providers where applicable in parentheses. TEE Technologies are colored in green while properties of the TEE are colored in yellow . These data points are examples to the process of comparison and should not be considered as a valid or current source of information. 


\begin{tabular}{|l|l|l|l|}
\hline & Intel SGX & Arm TrustZone & RISC-V PMP \\
\hline $\begin{array}{l}\text { Remote } \\
\text { Attestation }\end{array}$ & Intel dependent & $\begin{array}{l}\text { Platform } \\
\text { dependent } \\
\text { (Qualcomm) }\end{array}$ & Not available \\
\hline $\begin{array}{l}\text { Local } \\
\text { Attestation }\end{array}$ & Inherent in SGX & $\begin{array}{l}\text { Firmware } \\
\text { dependent } \\
\text { (TF-A) }\end{array}$ & $\begin{array}{l}\text { Firmware } \\
\text { dependent } \\
\text { (Keystone) }\end{array}$ \\
\hline $\begin{array}{l}\text { Hardware } \\
\text { RoT }\end{array}$ & $\begin{array}{l}\text { Platform } \\
\text { dependent (Intel, } \\
\text { Supermicro) }\end{array}$ & $\begin{array}{l}\text { Platform } \\
\text { dependent } \\
\text { (MCIMX8M- } \\
\text { EVKB) }\end{array}$ & $\begin{array}{l}\text { Platform } \\
\text { dependent } \\
\text { (MPFS-ICICLE- } \\
\text { KIT-ES) }\end{array}$ \\
\hline
\end{tabular}

Table 6.2: The possible attestation features and the dependencies of those features. Each technology provides local and remote attestation as a possibility. However, each technology requires that the consumer of the technology commit some level of effort into implementing their specific security model. Notably absent is remote attestation for RISC-V PMP. Remote attestation is indeed possible on these platforms, however no implementation is currently considered standard, and certainly no implementation is open source as of the writing of this thesis. TEE Technologies are colored in green while properties of the TEE are colored in yellow. These data points are examples to the process of comparison and should not be considered as a valid or current source of information.

${ }^{1}$ There is a RISC-V hardware root of trust that is available and fully open source, including an open source HDL implementation [28]. As an example platform one might use the Microchip PolarFire SoC Icicle kit (MPFS-ICICLE-KIT-ES). This platform contains non-volatile FPGA fabric which is made up of logic elements, on-chip memory, and math blocks. This is ideal for creating a hardware RoT. 


\begin{tabular}{|l|l|l|l|}
\hline & Intel SGX & Arm TrustZone & RISC-V PMP \\
\hline $\begin{array}{l}\text { Open Source } \\
\text { SDK }\end{array}$ & $\begin{array}{l}\text { Y (Intel SGX } \\
\text { SDK })\end{array}$ & Y (OP-TEE) & Y (Keystone) \\
\hline $\begin{array}{l}\text { Open Source } \\
\text { Firmware }\end{array}$ & $\begin{array}{l}\text { Y (TianoCore, } \\
\text { coreboot) }\end{array}$ & Y (TF-A) & Y (Keystone) \\
\hline $\begin{array}{l}\text { Extensible } \\
\text { HDL }\end{array}$ & N & Y & Y \\
\hline $\begin{array}{l}\text { Open Source } \\
\text { HDL }\end{array}$ & N & N & Y \\
\hline
\end{tabular}

Table 6.3: The possible extensibility features and the dependencies of those features. We consider open source options to be inherently extensible. Proprietary solutions are extensible only if modifications are supported by the vendor, as is the case with Arm and "extensible HDL". Certainly few if any Arm vendors will allow you to modify their HDL directly, however Arm's IP model does allow for some inherent modification by allowing one to pick and choose which IP they wish to use. Note that with Intel SGX, the concept of open source firmware will only allow limited extensibility to the functionality of the TEE. TEE Technologies are colored in green while properties of the TEE are colored in yellow . These data points are examples to the process of comparison and should not be considered as a valid or current source of information. 


\subsection{Considerations and Limitations}

In this comparison we have not distinguished between platform manufacturers and platforms vendors. As an example, Supermicro is a platform manufacturer which might produce a platform based on an Intel Xeon Processor currently code named "Ice Lake-SP" which will support SGX on multi-socket systems. They might then sell those platforms to a vendor like Hewlett Packard or Dell. How Intel SGX will function on these platforms becomes a combination of the instructions Intel has added to the architecture, the workflow inherent in the microarchitecture, how the chipset is assembled, how Supermicro assembled that chipset into a platform, and how HP or Dell supports that platform with firmware, software, and possibly remote attestation services. The same is true of Arm systems and RISC-V systems. One quickly sees the complexity of securing systems in the real world. Our method for comparison is meant only as a high level tool for assessing the appropriateness of a TEE technology to a use case.

The method we have described will also be limited by the scope of the use case. The complexity of the comparison will grow in a linear manner to the complexity of the use case. One can quickly postulate that a multi-party system based on heterogeneous TEEs will reach levels of complexity such that this method begins to break down, or at the very least requires much more rigor than the simple comparisons shown here. This method is submitted not as a golden standard for comparison, but rather as a baseline on which further research may provide avenues for comparison not imagined in this thesis. 


\section{Chapter 7}

\section{Conclusion}

This thesis has described a method that allows for a comprehensive and rigorous comparison of Trusted Execution Environments (TEEs). This method involves identifying the properties of interest for hardware architects, firmware authors, and TEE software designers alike. Key to our comparison of the implementations of those properties is breaking each property down into relevant constituent parts. The relevancy of each property will depend on the use case of the designer, and should be taken into account during comparison. This method does not provide us with which technology is the "best," but rather gives us insight into which TEE might best fit a specific set of needs.

There are several use cases for systems requiring a TEE that this thesis does not take into account. These use cases could be the subject of future work in improving this methodology. Multi-socket processors [34] add another layer of complexity to TEEs and should be considered for cloud compute or data center use cases. Likewise systems might require multiple TEEs or heterogeneous TEE environments, perhaps for enhanced security use cases. As an example, a system running both TrustZone alongside RISC-V Physical Memory Protection (PMP) may require communication between these different TEEs to assure secure computation. Examining the details of how these systems are able to interact and communicate allows for new avenues of comparison. Future work covering these use cases would add to the rigorous nature of this method.

This proposed method will save hardware architects the frustration that comes 
with a wide range of technology choices. The method provides an organizational framework for characterizing properties of a TEE and provides value to product designers who must focus on possible use cases ranging from mobile devices to cloud services. It is possible that this method may be insufficient for some future version of a TEE, as evidenced by how new technologies like RISC-V Physical Memory Protection (PMP) are both mimicking older technologies as well as developing new and innovative features. However, as the use of TEEs becomes more common and these technologies take on new unique properties, it is likely that these new properties will be easily integrated into this method of comparison. 


\section{References}

[1] I. Anati, S. Gueron, S. Johnson, and V. Scarlata, "Innovative technology for CPU based attestation and sealing," in Proceedings of the 2nd international workshop on hardware and architectural support for security and privacy, vol. 13. ACM New York, NY, USA, 2013, p. 7.

[2] P. Anemaet and T. van As, "Microprocessor soft-cores: An evaluation of design methods and concepts on FPGAs," part of the Computer Architecture (Special Topics) course ET4078, Department of Computer Engineering, 2003.

[3] Arm Limited, "Arm Security Technology Building a Secure System using TrustZone Technology, Issue C," Arm Limited, Tech. Rep., April 2009.

[4] — , "Fundamentals of ARMv8-A Version 1.0," Arm Limited, Tech. Rep., March 2015.

[5] — , "Trusted Base System Architecture, Client, Version F," Arm Limited, Tech. Rep., August 2020.

[6] Arm Limited, Architecture and Technology Group, "Trusted Board Boot Requirements CLIENT (TBBR-CLIENT) ARMv8-A, Version 1.0," Arm Limited, Tech. Rep., September 2018.

[7] K. Asanovic, R. Avizienis, J. Bachrach, S. Beamer, D. Biancolin, C. Celio, H. Cook, D. Dabbelt, J. Hauser, A. Izraelevitz et al., "The rocket chip generator," EECS Department, University of California, Berkeley, Tech. Rep. $U C B / E E C S-2016-17,2016$. 
[8] K. Asanović and D. A. Patterson, "Instruction sets should be free: The case for RISC-V," EECS Department, University of California, Berkeley, Tech. Rep. UCB/EECS-2014-146, 2014.

[9] J. G. Beekman, "Improving cloud security using secure enclaves," Ph.D. dissertation, UC Berkeley, 2016.

[10] T. Bourgeat, I. Lebedev, A. Wright, S. Zhang, and S. Devadas, "Mi6: Secure enclaves in a speculative out-of-order processor," in Proceedings of the 52nd Annual IEEE/ACM International Symposium on Microarchitecture, 2019, pp. $42-56$.

[11] E. Brickell and J. Li, "Enhanced privacy ID: A direct anonymous attestation scheme with enhanced revocation capabilities," in Proceedings of the 2007 ACM workshop on Privacy in electronic society, 2007, pp. 21-30.

[12] G. Cabodi, P. Camurati, C. Loiacono, G. Pipitone, F. Savarese, and D. Vendraminetto, "Formal verification of embedded systems for remote attestation," WSEAS Transactions on Computers, vol. 14, pp. 760-769, 2015.

[13] S. Chakrabarti, M. Hoekstra, D. Kuvaiskii, and M. Vij, "Scaling Intel Software Guard Extensions Applications with Intel SGX Card," in Proceedings of the 8th International Workshop on Hardware and Architectural Support for Security and Privacy, ser. HASP '19. New York, NY, USA: Association for Computing Machinery, 2019. [Online]. Available: https://doi.org/10.1145/3337167.3337173 
[14] K. Cheang, C. Rasmussen, D. Lee, D. W. Kohlbrenner, K. Asanovic, and S. A. Seshia, "Verifying RISC-V physical memory protection," IEEE International Symposium on Performance Analysis of Systems and Software (ISPASS) Workshop on Secure RISC-V Architecture Design, August 2020.

[15] V. Costan and S. Devadas, "Intel SGX Explained," International Association for Cryptologic Research ePrint Archive, pp. 1-118, 2016. [Online]. Available: http://eprint.iacr.org/2016/086

[16] V. Costan, I. Lebedev, and S. Devadas, "Sanctum : Minimal Hardware Extensions for Strong Software Isolation This paper is included in the Proceedings of the Sanctum : Minimal Hardware Extensions for Strong Software Isolation," Usenix, vol. 25, pp. 857-874, 2016. [Online]. Available: https://www.usenix.org/conference/usenixsecurity16/technicalsessions/presentation/costan

[17] M. Da Silva, "Securing a trusted hardware environment (trusted execution environment)," Ph.D. dissertation, Université Montpellier, 2018.

[18] B. Delgado and K. L. Karavanic, "Performance implications of system management mode," in 2013 IEEE International Symposium on Workload Characterization (IISWC), 2013, pp. 163-173.

[19] D. Eldar, A. Kumar, and P. Goel, "Configuring Intel active management technology." Intel Technology Journal, vol. 12, no. 4, 2008.

[20] K. Eldefrawy, N. Rattanavipanon, and G. Tsudik, "Hydra: hybrid design for remote attestation (using a formally verified microkernel)," in Proceedings of 
the 10th ACM Conference on Security and Privacy in wireless and Mobile Networks, 2017, pp. 99-110.

[21] T. Garfinkel, B. Pfaff, J. Chow, M. Rosenblum, and D. Boneh, "Terra: A virtual machine-based platform for trusted computing," in Proceedings of the nineteenth ACM symposium on Operating systems principles, 2003, pp. 193206.

[22] C. Gentry, "A fully homomorphic encryption scheme," Ph.D. dissertation, Stanford University, Stanford, CA, USA, 2009.

[23] GlobalPlatform Inc, "GlobalPlatform TEE Client API Specification Version 1.0," 072010.

[24] — _ "GlobalPlatform TEE Client API Specification Version 1.0," 072010.

[25] — _ "GlobalPlatform Technology TEE System Architecture Version 1.2," 11 2018.

[26] — - "GlobalPlatform TEE Internal Core API Specification Version 1.2.1," 052019.

[27] — , "GlobalPlatform TEE Protection Profile Version 1.3," 092020.

[28] S. Guilley, M. Le Rolland, and D. Quenson, "Implementing secure applications thanks to an integrated secure element," in 7th International Conference on Information Systems Security and Privacy, 2021.

[29] J. Haj-Yahya, M. M. Wong, V. Pudi, S. Bhasin, and A. Chattopadhyay, "Lightweight secure-boot architecture for RISC-V system-on-chip," in 20th 
International Symposium on Quality Electronic Design (ISQED). IEEE, 2019, pp. 216-223.

[30] M. Hoekstra, R. Lal, P. Pappachan, V. Phegade, and J. Del Cuvillo, "Using innovative instructions to create trustworthy software solutions," in Proceedings of the 2nd International Workshop on Hardware and Architectural Support for Security and Privacy, ser. HASP '13. New York, NY, USA: Association for Computing Machinery, 2013. [Online]. Available: https://doi.org/10.1145/2487726.2488370

[31] S. Johnson, V. Scarlata, C. Rozas, E. Brickell, and F. Mckeen, "Intel software guard extensions: EPID provisioning and attestation services," White Paper, vol. 1, no. 1-10, p. 119, 2016.

[32] S. P. Johnson, U. R. Savagaonkar, V. R. Scarlata, F. X. McKeen, and C. V. Rozas, "Technique for supporting multiple secure enclaves," Dec. 2010, US Patent 8,972,746.

[33] P. A. Karger and A. J. Herbert, "An augmented capability architecture to support lattice security and traceability of access," in 1984 IEEE Symposium on Security and Privacy, 1984, pp. 2-2.

[34] T. Knauth, M. Steiner, S. Chakrabarti, L. Lei, C. Xing, and M. Vij, "Integrating remote attestation with transport layer security," arXiv preprint $\operatorname{arXiv:1801.05863,2018.}$

[35] I. Lebedev, K. Hogan, and S. Devadas, "Secure boot and remote attestation in the sanctum processor," in 2018 IEEE 31st Computer Security Foundations Symposium (CSF). IEEE, 2018, pp. 46-60. 
[36] D. Lee, D. Kohlbrenner, S. Shinde, K. Asanović, and D. Song, "Keystone: An open framework for architecting trusted execution environments," in Proceedings of the Fifteenth European Conference on Computer Systems, 2020, pp. $1-16$.

[37] D. Lee, D. Kohlbrenner, S. Shinde, D. Song, and K. Asanović, "Keystone: A Framework for Architecting TEEs," CoRR, vol. abs/1907.10119, 2019. [Online]. Available: http://arxiv.org/abs/1907.10119

[38] Linaro Limited. Trusted Firmware. [Online]. Available: https: //www.trustedfirmware.org

[39] lowRISC. Ibex Core. [Online]. Available: https://github.com/lowRISC/ibex

[40] D. W. Manchala, "Trust metrics, models and protocols for electronic commerce transactions," in Proceedings. 18th International Conference on Distributed Computing Systems (Cat. No.98CB36183), 1998, pp. 312-321.

[41] F. X. McKeen, C. V. Rozas, U. R. Savagaonkar, S. P. Johnson, V. Scarlata, M. A. Goldsmith, E. Brickell, J. T. Li, H. C. Herbert, P. Dewan et al., "Method and apparatus to provide secure application execution," Dec. 2009, US Patent $9,087,200$.

[42] F. McKeen, I. Alexandrovich, A. Berenzon, C. V. Rozas, H. Shafi, V. Shanbhogue, and U. R. Savagaonkar, "Innovative instructions and software model for isolated execution," in Proceedings of the 2nd International Workshop on Hardware and Architectural Support for Security and Privacy, ser. HASP '13. New York, NY, USA: Association for Computing Machinery, 2013. [Online]. Available: https://doi.org/10.1145/2487726.2488368 
[43] M. Nauman, S. Khan, X. Zhang, and J.-P. Seifert, "Beyond kernel-level integrity measurement: enabling remote attestation for the android platform," in International Conference on Trust and Trustworthy Computing. Springer, 2010, pp. 1-15.

[44] B. Ngabonziza, D. Martin, A. Bailey, H. Cho, and S. Martin, "TrustZone explained: Architectural features and use cases," in 2016 IEEE 2nd International Conference on Collaboration and Internet Computing (CIC), 2016, pp. $445-451$.

[45] Open Mobile Terminal Platform, "OMTP: Advanced Trusted Environment: OMTP TR1," Open Mobile Terminal Platform, Tech. Rep., 012009.

[46] T. O’Reilly, "Lessons from open-source software development," Communications of the ACM, vol. 42, no. 4, pp. 32-37, 1999.

[47] J. D. Osborn and D. C. Challener, "Trusted platform module evolution," Johns Hopkins APL Technical Digest (Applied Physics Laboratory), vol. 32, no. 2, pp. 536-543, 2013.

[48] S. Perez. WAC Whacked: Telecom-Backed Alliance Merges Into GSMA, Assets Acquired By API Management Service Apigee. [Online]. Available: https://techcrunch.com/2012/07/17/wac-whacked-telecom-backed-alliancemerges-into-gsma-assets-acquired-by-api-management-service-apigee

[49] A. S. Perrin, "The general data protection regulation and open source software communities," CybarisßR: Vol. 12 : Iss. 1 , Article 3, 2021.

[50] S. Pinto and J. Martins, "The industry-first secure IoT stack for RISC-V: a research project," in RISC-V Workshop,(Zurich), 2019. 
[51] S. Pinto and N. Santos, "Demystifying Arm TrustZone: A Comprehensive Survey," ACM Computing Surveys, vol. 51, no. 6, 2019.

[52] E. Raymond, "The cathedral and the bazaar," Knowledge, Technology \& Policy, vol. 12, no. 3, pp. 23-49, 1999.

[53] D. M. Rodriguez, D. H. Aristizabal, and R. Y. Guevara, "A primer to the token stealing technique," in 2013 47th International Carnahan Conference on Security Technology (ICCST), 2013, pp. 1-3.

[54] M. Sabt, M. Achemlal, and A. Bouabdallah, "Trusted execution environment: What it is, and what it is not," in 2015 IEEE Trustcom/BigDataSE/ISPA, vol. 1, 2015, pp. 57-64.

[55] M. Schwarz, S. Weiser, and D. Gruss, "Practical enclave malware with Intel SGX," in International Conference on Detection of Intrusions and Malware, and Vulnerability Assessment. Springer, 2019, pp. 177-196.

[56] C. Shepherd, K. Markantonakis, and G.-A. Jaloyan, "LIRA-V: Lightweight remote attestation for constrained RISC-V devices," arXiv preprint arXiv:2102.08804, 2021.

[57] C. Spensky, J. Stewart, A. Yerukhimovich, R. Shay, A. Trachtenberg, R. Housley, and R. K. Cunningham, "Sok: Privacy on mobile devices-it's complicated," Proceedings on Privacy Enhancing Technologies, vol. 2016, no. 3, pp. 96-116, 2016.

[58] Technical Advisory Council, "A Technical Analysis of Confidential Computing Version 1.1," Confidential Computing Consortium, Tech. Rep., 012021. 
[59] F. Teschke, "Hardening Applications with Intel SGX," Master's thesis, HassoPlattner-Institut at the University of Potsdam, Prof.-Dr.-Helmert-Straße 2-3, 14482 Potsdam, Germany, June 2017, Anwendungen härten mit Intel SGX.

[60] UEFI Forum, "Unified Extensible Firmware Interface (UEFI) Specification Version 2.8 Errata B," May 2020.

[61] R. Uhlig, G. Neiger, D. Rodgers, A. L. Santoni, F. C. Martins, A. V. Anderson, S. M. Bennett, A. Kagi, F. H. Leung, and L. Smith, "Intel virtualization technology," Computer, vol. 38, no. 5, pp. 48-56, 2005.

[62] A. Waterman and K. Asanović, "The RISC-V Instruction Set Manual, Volume I: User-Level ISA, Document Version 20191213," RISC-V ISA, vol. I, 2019. [Online]. Available: https://github.com/riscv/riscv-isa-manual/ releases/download/Ratified-IMAFDQC/riscv-spec-20191213.pdf

[63] —, "The RISC-V Instruction Set Manual, Volume II: Privileged Architecture, Document Version 20190608," RISC-V ISA, vol. II, 2019. [Online]. Available: https://github.com/riscv/riscv-isa-manual/releases/ download/Ratified-IMFDQC-and-Priv-v1.11/riscv-privileged-20190608.pdf

[64] N. Weichbrodt, A. Kurmus, P. Pietzuch, and R. Kapitza, "AsyncShock: Exploiting synchronisation bugs in Intel SGX enclaves," in European Symposium on Research in Computer Security. Springer, 2016, pp. 440-457.

[65] S. Weiser, M. Werner, F. Brasser, M. Malenko, S. Mangard, and A.-R. Sadeghi, "TIMBER-V: Tag-isolated memory bringing fine-grained enclaves to RISC-V." in NDSS, 2019. 
[66] J. Yao, V. J. Zimmer, and Q. Long, "System management mode isolation in firmware," May 7 2009, US Patent App. 12/317,446.

[67] V. Zimmer, "Hardened extensible firmware framework to support system management mode operations using 64-bit extended memory mode processors," May 26 2005, US Patent App. 10/971,824.

[68] V. Zimmer, M. Rothman, and S. Marisetty, Beyond BIOS: Developing with the Unified Extensible Firmware Interface, Third Edition, 3rd ed. Berlin, DEU: De|G Press, 2017.

[69] V. J. Zimmer, M. Kumar, M. Natu, Q. Long, L. Cui, and J. Yao, "Apparatus and method for secure boot environment," Jul. 19 2011, US Patent 7,984,286. 